\title{
A contact smoothing method for arbitrary surface meshes using Nagata patches
}

\author{
D.M. Neto $^{\text {a,* }}$, M.C. Oliveira ${ }^{\text {a }}$, L.F. Menezes ${ }^{a}$, J.L. Alves ${ }^{b}$

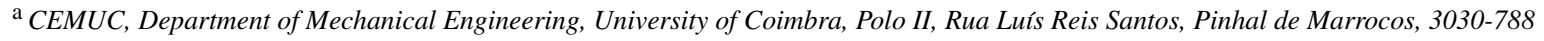 \\ Coimbra, Portugal \\ ${ }^{\mathrm{b}}$ MEMS, Department of Mechanical Engineering, University of Minho, Campus de Azurém, 4800-058 Guimarães, Portugal
}

Received 5 January 2015; received in revised form 20 July 2015; accepted 6 November 2015

Available online 14 November 2015

\section{Highlights}

- A new 3D contact surface smoothing approach for large deformation contact problems between deformable bodies is proposed.

- The local Nagata patch interpolation is used to smooth arbitrary surface meshes.

- The original curvature of the master surface is recovered using a relatively coarse mesh.

- The non-physical contact force oscillations usual in the faceted surface representation are eliminated.

- The accuracy, robustness and performance of the numerical simulations is improved adopting the surface smoothing method.

\begin{abstract}
This paper presents a contact surface smoothing method combined with the node-to-segment discretization technique to solve large deformation frictional contact problems between deformable bodies. The Nagata patch interpolation is used to smooth the surface mesh, providing a master surface with quasi- $G^{1}$ continuity between patches. Moreover, the local support of the interpolation method allows to deal with surface meshes of arbitrary topology (regular and irregular finite element discretizations), as well as hybrid meshes. The non-physical oscillations in the contact force evolution, induced by the faceted contact surface representation, are reduced using the proposed smoothing method. Furthermore, the smooth representation of the master surface allows a more accurately evaluation of the resulting stresses and forces, while providing an important improvement in convergence behaviour. Four representative numerical examples are used to demonstrate the advantages of the proposed contact smoothing method. The results show a significant improvement in the accuracy, robustness and performance of the numerical simulations using the smoothing approach, when compared with the piecewise faceted contact surface description.
\end{abstract}

(C) 2015 Elsevier B.V. All rights reserved.

Keywords: Finite element method; Frictional contact; Large sliding; Surface smoothing; Nagata patch; Augmented Lagrangian method

\footnotetext{
* Correspondence to: Department of Mechanical Engineering, University of Coimbra, Rua Luís Reis Santos, Pinhal de Marrocos, 3030-788 Coimbra, Portugal. Tel.: +351 239790700; fax: +351 239790701 .

E-mail addresses: diogo.neto@ dem.uc.pt (D.M. Neto), marta.oliveira@dem.uc.pt (M.C. Oliveira), luis.menezes@ dem.uc.pt (L.F. Menezes), jlalves@dem.uminho.pt (J.L. Alves).
} 


\section{Introduction}

The description of the contact interaction across bodies plays an important role in many engineering problems. However, the numerical simulation of frictional contact between solids undergoing large deformations using implicit methods is still one of the most challenging tasks in computational mechanics, due to the highly nonlinear and nonsmooth behaviour [1,2]. Indeed, the inequality constraints resulting from the impenetrability condition and the friction law are expressed by non-smooth multivalued relationships. The approaches usually considered for incorporating the contact constraints in the variational formulation of the equilibrium problem are: (i) the penalty method [3-6]; (ii) the Lagrange multiplier method [7-9] and (iii) the augmented Lagrangian method [10-12]. The penalty method is widely used due to its simple formulation, although the adequate choice of the penalty parameter may be difficult [13]. In fact, low values of the penalty parameter lead to the inaccurate enforcement of the contact constraint conditions (unacceptable penetration), while high values of the penalty parameters can lead to the ill-conditioning of the stiffness matrix. The Lagrange multiplier method exactly enforces the impenetrability and friction constraints, introducing extra variables (Lagrange multipliers), which represent the contact forces. The augmented Lagrangian method takes advantage of these two cited methods, allowing the exact representation of the contact constraints for a finite value of the penalty parameter. The generalized Newton method can be applied to solve the mixed system of equations (displacements and Lagrange multipliers as unknowns) [10,14], or alternatively the solution can be obtained with the Uzawa's algorithm [12], where the unknowns are only the displacements due to the nested update of dual variables (Lagrange multipliers).

The discretization of the contact interface in problems involving large sliding between deformable bodies is commonly performed with the node-to-segment (NTS) contact algorithm developed by Hallquist [4]. It is combined with the master-slave approach, where the enforcement of the contact constraints (impenetrability and friction conditions) is established in the nodes of the slave surface, preventing its penetration in the opposing discretized master surface. Since the geometry of the contacting surfaces is arbitrarily curved, its spatial discretization with low order finite elements introduces discontinuities in the surface normal vector field (facetization problem) [15]. Indeed, the bilinear surface facets defining the master surface are created using the exterior nodes of the low order solid elements defining the solid body. This geometric discontinuity leads to numerical instability, loss of the quadratic convergence rate in the non-linear solution scheme and non-physical oscillations in the contact force when a slave node slides over several master facets [16].

In order to overcome the chatter effect induced by the spatial discretization, several surface smoothing procedures have been proposed in the context of NTS formulation. Since the kinematic constraints are more accurately evaluated (the gap function and the surface normal vector), the robustness of the contact algorithms and the accuracy of the solution is significantly improved adopting a smoothing scheme [15,17-19]. In the NTS formulation only the master surface is smoothed, creating parametric patches over the discretized surface using the coordinates of the master nodes, dictating that the slave nodes interact with a smooth master surface. Different interpolation methods have been applied to smooth the contact surface mesh of deformable bodies: cubic Hermite interpolation [17], cubic Bézier [16,20], cubic Splines [21,22] and NURBS [23,24]. All these approaches were originally developed for 2D problems, thus its extension to describe contact surfaces in 3D is restricted to regular quadrilateral meshes, since the patches are obtained using the tensor product. In fact, the application of a smoothing method to arbitrary surface meshes is more difficult, because the number of neighbouring facets taken into account to generate the interpolated surface is arbitrary [25]. Only two approaches are available to deal with irregular 3D surface meshes. The first one, proposed by Puso and Laursen [26], uses Gregory patches in the surface smoothing, providing $G^{1}$ continuity between adjacent patches. It can be applicable to both regular and irregular meshes of quadrilateral facets. The other approach, developed by Krstulovic-Opara et al. [27], employs quartic Bézier patches in the interpolation using the nodes and the centroid of triangular finite elements. This approach leads to $C^{1}$ continuity everywhere except at the element nodes. On the other hand, the approach proposed by Belytschko et al. [28], is an alternative to the classic surface smoothing methods, performing the smoothing implicitly by constructing smooth signed distance functions from a scattered set of nodes, using a moving least-squares approximation. Although this method can be applicable to arbitrary surface meshes, the generated smoothed surface does not pass through the master nodes exactly, which can introduce some inaccuracies in the contact geometry [29]. Assuming that one contact body is rigid (Signorini problems), various computer aided design (CAD) interpolations can be used to define 3D smooth surfaces [30-32]. Nevertheless, this corresponds to a simpler problem than the case of two deformable bodies, since the master surface cannot be deformed. 
a

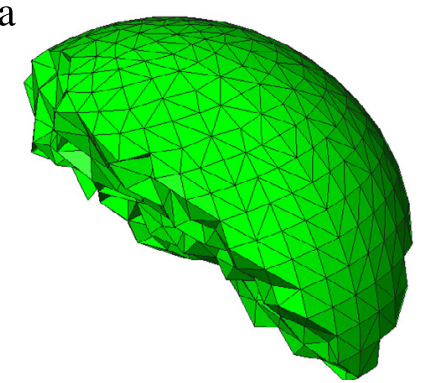

b

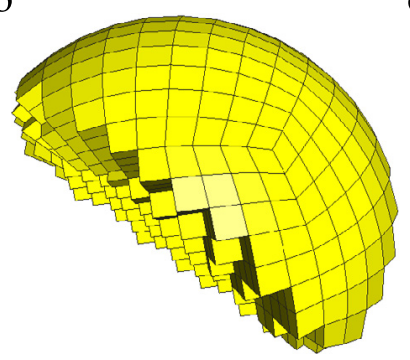

$\mathrm{c}$

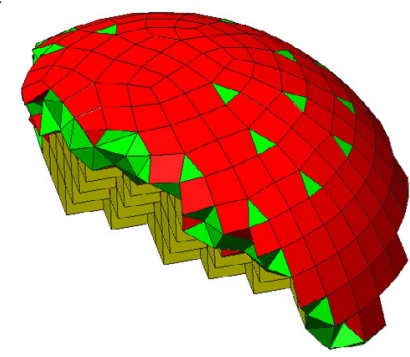

Fig. 1. Different types of finite elements used for the spatial discretization of a sphere: (a) linear tetrahedral element mesh; (b) linear hexahedral element mesh; (c) hybrid mesh composed by tetrahedral, hexahedral and pyramidal elements.

In the last years, the segment-to-segment formulation [33] coupled with the mortar method has been successfully applied to solve large deformation frictional contact problems [34-38], overcoming some well-known drawbacks exhibited by the classical NTS formulation, such as the chatter effect in the contact force evolution. Since the contact constraints are imposed in the weak form, using integrals defined in the entire discretized contact area, the mortar method contains inherent smoothing. The transmission of forces is performed through its distribution in the whole contact surface, allowing to satisfy the contact patch test introduced by Taylor and Papadopoulos [39], i.e. exactly transfer a constant pressure through a flat surface. Although the classical NTS contact formulation fails the contact patch test using the single pass algorithm [39], applying the two pass algorithm in conjunction with the Lagrange multiplier method allows to satisfy the patch test for low order finite elements [40,41]. Concurrently, significant effort has been made in recent years to develop the isogeometric analysis for solving contact problems [42-44], firstly introduced by Hughes et al. [45]. Since the parameterization of both the geometry and the displacement field is based on basis functions emanating from the CAD (e.g. NURBS) rather than on Lagrange polynomial elements, the advantages for modelling contact problems is evident. The contact force oscillations arising in large sliding contact when using conventional Lagrange polynomial elements are effectively alleviate, yielding highly robust schemes due to the continuous smooth surface approximation [46]. Nevertheless, its application in contact problems involving complex geometries requires a careful construction of the CAD model in order to avoid trimmed NURBS surfaces.

The spatial discretization of a simple sphere using different types of finite elements is presented in Fig. 1, establishing the typology of the contact surface mesh. The discretization of complex geometries with tetrahedral finite elements (Fig. 1(a)) is significantly easier than with hexahedral elements (Fig. 1(b)), since it is possible to use automatic meshing tools [47]. On the other hand, hybrid meshes overcome the main drawback of regular meshes (lack of flexibility), while combining the advantages of regular and irregular meshes [48]. The sphere presented in Fig. 1(c) is discretized with a regular mesh of hexahedral elements in the interior of the volume and pyramidal elements at the interface between hexahedral and tetrahedral finite elements. The surface contact mesh is composed mainly by pyramidal elements and some tetrahedral finite elements, leading to a hybrid surface mesh. Regarding the smoothing of irregular surface meshes, the smoothing method developed by Krstulovic-Opara et al. [27] is restricted to triangular facets derived from the 4-node tetrahedral finite elements (Fig. 1(a)), while the approach proposed by Puso and Laursen [26] is limited to quadrilateral facets resulting from the 8-node hexahedral finite elements (Fig. 1(b)).

The purpose of the present study is to develop a contact surface smoothing procedure for arbitrary 3D surface meshes. The Nagata patch interpolation [49] is adopted for the smooth representation of the master surface, where each patch is created using only the position and surface normal vectors at the nodes of each facet. Indeed, the local support of the interpolation method allows to deal with hybrid surface meshes of arbitrary topology (irregular meshes composed by triangular and quadrilateral facets), which is the main feature of the proposed surface smoothing procedure. This interpolation method was previously applied to smooth rigid surfaces involved in 3D contact problems [50-52]. This work presents the extension of this interpolation method to deal with contact problems between deformable bodies, where the smoothed surface will suffer large deformations.

Following this introductory section, the governing equations of the frictional contact problem between two deformable bodies undergoing large deformation are introduced in Section 2, using the augmented Lagrangian approach to impose the contact constraints. The Nagata patch interpolation method is reviewed in Section 3, followed by the description of the proposed contact surface smoothing procedure, which is compared with the traditional 


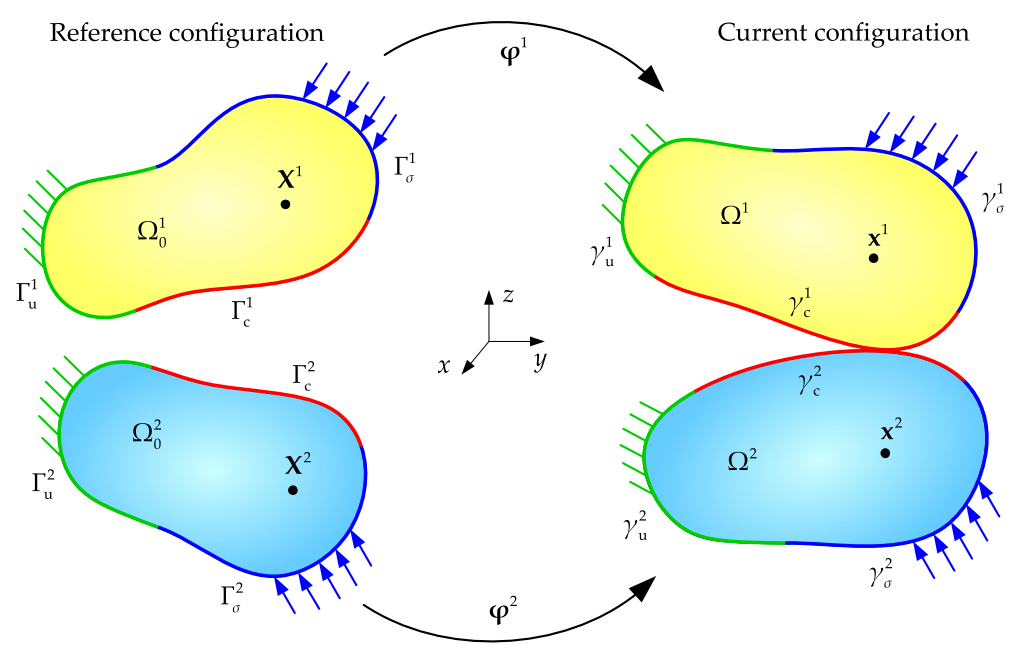

Fig. 2. Notation for the two body large deformation frictional contact problem.

piecewise faceted representation. The formulation of the node-to-Nagata contact elements (triangular and quadrilateral Nagata patch) used to deal with large sliding frictional contact problems is presented in Section 4, including the definition of the residual vectors and tangent matrices. Four representative numerical examples are presented in Section 5, illustrating the accuracy and effectiveness of the proposed contact surface smoothing method. Finally, the main conclusions of this study are discussed in Section 6.

\section{Contact mechanics problem}

The 3D frictional contact problem between two deformable bodies undergoing large deformation is briefly reviewed in the continuum framework, following the notation adopted by Laursen and Simo [53]. Without loss of generality, for purposes of simplicity, the contact problem between two bodies $\mathcal{B}^{\alpha}(\alpha=1,2)$ defined within the Euclidean space $\mathbb{R}^{3}$ is considered, as illustrated in Fig. 2. In case of large deformation, it is necessary to distinguish between the reference and the current configurations. The contacting bodies in the reference configuration are represented by the open sets $\Omega_{0}^{\alpha} \subset \mathbb{R}^{3}$, while their boundaries are denoted by $\partial \Omega_{0}^{\alpha}$. The union of the open set with its boundary is denoted by $\bar{\Omega}_{0}^{\alpha}=\Omega_{0}^{\alpha} \cup \partial \Omega_{0}^{\alpha}$ for each body. The deformation mappings of the bodies are denoted by $\boldsymbol{\phi}^{\alpha}$, for some closed time interval of interest $t \in[0, T]$. The material points of each body are denoted by $\mathbf{X}^{\alpha} \in \bar{\Omega}_{0}^{\alpha}$ in the reference configuration and by $\mathbf{x}^{\alpha}=\boldsymbol{\phi}^{\alpha}\left(\mathbf{X}^{\alpha}, t\right)$ in the current configuration $\Omega^{\alpha}$ at time $t$, as shown in Fig. 2. The vector connecting any point of body $\mathcal{B}^{\alpha}$ in its current configuration with the position of the same point in the reference configuration is called displacement vector $\mathbf{u}^{\alpha}=\mathbf{x}^{\alpha}-\mathbf{X}^{\alpha}$. The surfaces of the bodies in the current configuration, denoted by $\partial \Omega^{\alpha}$, are divided into three non-overlapping regions: $\gamma_{\mathrm{u}}^{\alpha}$ where displacements are prescribed (Dirichlet boundary conditions), $\gamma_{\sigma}^{\alpha}$ where tractions are prescribed (Neumann boundary conditions) and $\gamma_{\mathrm{c}}^{\alpha}$ where the frictional contact constraints are defined (see Fig. 2). The spatial counterparts of these three areas are denoted by $\Gamma_{\mathrm{u}}^{\alpha}, \Gamma_{\sigma}^{\alpha}$ and $\Gamma_{\mathrm{c}}^{\alpha}$, respectively. Although the identification of the master and slave body is somewhat arbitrary, the bodies $\mathcal{B}^{1}$ and $\mathcal{B}^{2}$ will be referenced as slave and master, respectively. Using the same terminology for their boundaries, the contact surfaces $\gamma_{c}^{1}$ and $\gamma_{c}^{2}$ will be denoted as slave and master surfaces, respectively.

Assuming quasi-static response, the equilibrium equations and the boundary conditions for each body within the large deformation framework in absence of contact are given as follows:

$$
\begin{cases}\operatorname{div}\left(\boldsymbol{\sigma}^{\alpha}\right)+\mathbf{b}^{\alpha}=\mathbf{0} & \text { in } \Omega^{\alpha} \\ \mathbf{t}^{\alpha}=\boldsymbol{\sigma}^{\alpha} \mathbf{n}^{\alpha}=\overline{\mathbf{t}}^{\alpha} & \text { on } \gamma_{\sigma}^{\alpha} \\ \mathbf{u}^{\alpha}=\overline{\mathbf{u}}^{\alpha} & \text { on } \gamma_{\mathbf{u}}^{\alpha}\end{cases}
$$

where $\boldsymbol{\sigma}^{\alpha}$ denotes the Cauchy stress tensor, $\mathbf{b}^{\alpha}$ stands for the body force per unit current volume, $\mathbf{t}^{\alpha}$ represents the Cauchy traction vector (force per unit surface area in the current configuration) and $\mathbf{n}^{\alpha}$ is the outward unit normal vector to the boundary $\partial \Omega^{\alpha}$. The prescribed Cauchy traction vectors and the prescribed displacements over the 


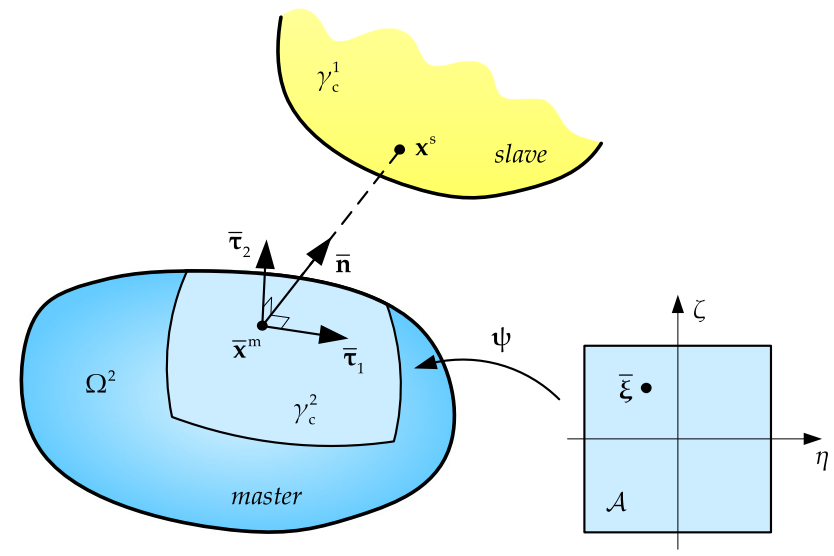

Fig. 3. Definition of the closest point projection in the current configuration, including the parameterization of the master surface.

indicated regions are denoted by $\overline{\mathbf{t}}^{\alpha}$ and $\overline{\mathbf{u}}^{\alpha}$, respectively. The divergence operator $\operatorname{div}(\bullet)$ involved in (1) is defined with respect to the spatial coordinates (current configuration).

\subsection{Kinematic contact constraints}

In order to distinguish between the points located in the interior of the bodies and the points placed on the contact surfaces, $\mathbf{x}^{\mathrm{s}} \in \gamma_{\mathrm{c}}^{1}$ and $\mathbf{x}^{\mathrm{m}} \in \gamma_{\mathrm{c}}^{2}$ refer to the slave and master points, respectively. It is useful to parameterize the master surface by defining $\mathcal{A} \in \mathbb{R}^{2}$ and a mapping $\psi: \mathcal{A} \rightarrow \mathbb{R}^{3}$ such that $\mathbf{x}^{\mathrm{m}}=\psi(\boldsymbol{\xi})$, where $\boldsymbol{\xi}=(\eta, \zeta) \in \mathcal{A}$ denotes the parameterization of $\gamma_{\mathrm{c}}^{2}$ via convective coordinates, as illustrated in Fig. 3.

The motion of the slave body with respect to the master body is defined adopting the master-slave approach, leading to an asymmetry in the definition of the contact problem. Assuming that the master surface is locally convex, each slave point $\mathbf{x}^{\mathrm{s}}$ on the surface $\gamma_{\mathrm{c}}^{1}$ can be related to a point $\overline{\mathbf{x}}^{\mathrm{m}}=\mathbf{x}^{\mathrm{m}}(\bar{\eta}, \bar{\zeta})$ belonging to the master surface $\gamma_{\mathrm{c}}^{2}$ using the following minimization distance problem:

$$
\left\|\mathbf{x}^{\mathrm{s}}-\overline{\mathbf{x}}^{\mathrm{m}}\right\|=\min _{\mathbf{x}^{\mathrm{m}} \in \gamma_{\mathrm{c}}^{2}}\left\|\mathbf{x}^{\mathrm{s}}-\mathbf{x}^{\mathrm{m}}\left(\xi^{1}, \xi^{2}\right)\right\|,
$$

where $\overline{\mathbf{x}}^{\mathrm{m}}$ is the closest master point to the slave point $\mathbf{x}^{\mathrm{s}}$, as shown in Fig. 3. This point is obtained from the normal projection of the slave point onto the master surface [54]. All geometric quantities evaluated at the closest projection point are denoted by a bar over the quantity. Therefore, the normal gap function is given by:

$$
g_{\mathrm{n}}=\left(\mathbf{x}^{\mathrm{s}}-\overline{\mathbf{x}}^{\mathrm{m}}\right) \cdot \overline{\mathbf{n}},
$$

where the unit normal vector of the master surface at the projection point $\overline{\mathbf{x}}^{\mathrm{m}}$ is denoted by $\overline{\mathbf{n}}=\mathbf{n}(\bar{\eta}, \bar{\zeta})$. Since only the master surface is parameterized, the superscript $\mathbf{n}^{2}$ is omitted for convenience. The sign of the normal gap function (3) provides the geometrical status of the slave point, which is positive if the contact is open and negative when penetration of the bodies takes place [10]. The balance of the linear momentum defined across the contact interface dictates that the contact force exerted on the master body $\mathcal{B}^{2}$ is equal and opposite to the force applied on the slave body $\mathcal{B}^{1}$. Hence, the action-reaction principle expresses a relationship between the Cauchy contact traction in each body, defined by $\mathbf{t}^{2}(\eta, \zeta)=-\mathbf{t}^{1}=\mathbf{t}$ at the contact point $\overline{\mathbf{x}}^{\mathrm{m}}$. Since the frictional response at the contact interface is taken into account, the Cauchy contact traction must be decomposed into the normal and tangential components, as follows:

$$
\mathbf{t}=p_{\mathrm{n}} \overline{\mathbf{n}}+\mathbf{t}_{\mathbf{t}}
$$

where the contact pressure is calculated by $p_{\mathrm{n}}=\mathbf{t} \cdot \overline{\mathbf{n}}$ and the tangential component is evaluated by $\mathbf{t}_{\mathrm{t}}=(\mathbf{I}-\overline{\mathbf{n}} \otimes \overline{\mathbf{n}}) \mathbf{t}$. From the physical point of view, the Cauchy contact traction defined in (4) represents the contact force applied by the slave body $\mathcal{B}^{1}$ on the master body $\mathcal{B}^{2}$, at the contact point $\overline{\mathbf{x}}^{\mathrm{m}}$. 


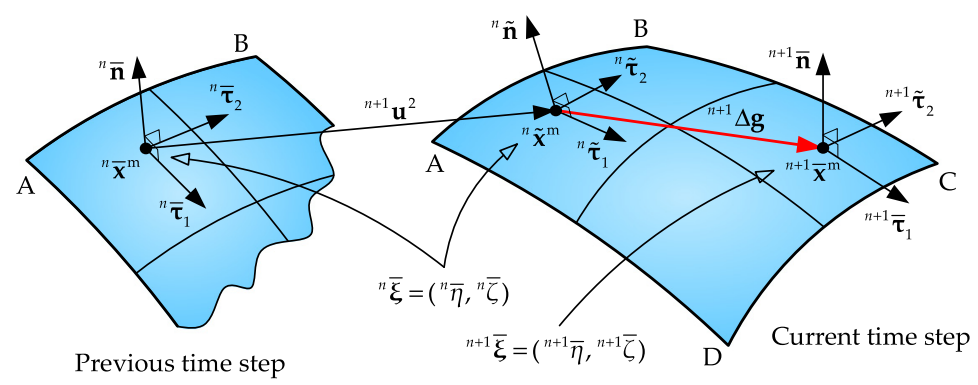

Fig. 4. Definition of the tangential slip vector using the mapping of the projection point from the previous time step forward to the current time step.

The unilateral contact law enforces the physical requirement of impenetrability and compressive interaction between contact bodies, summarized by the Karush-Kuhn-Tucker (KKT) conditions as follows:

$$
g_{\mathrm{n}} \geq 0 ; \quad p_{\mathrm{n}} \leq 0 ; \quad g_{\mathrm{n}} p_{\mathrm{n}}=0,
$$

where the first condition expresses the impenetrability between the bodies, the second imposes that contact pressure (normal component of the Cauchy contact traction) is compressive, while the third condition states a complementarity condition, imposing that $g_{\mathrm{n}}$ and $p_{\mathrm{n}}$ cannot be simultaneously non-null.

Several models have been developed to describe the friction behaviour [2,55]. Nevertheless the simple nonassociated Coulomb friction law is adopted in the present study. Thus, the relative tangential slip between the contacting bodies must be introduced. It is related to the change of the solution $(\bar{\eta}, \bar{\zeta})$ obtained for the closest point projection (2), providing the path of the slave point over the master surface. The local parameterization of the master surface induced by the finite element discretization leads to difficulties in the evaluation of the tangential slip, particularly for irregular finite element meshes. When the incremental slip path of the slave node comprises several finite elements (large sliding) [26], the time integration of the convective coordinates variation becomes meaningless [53]. This problem can be avoided using the history information, as described by Agelet de Saracibar [56], where the slip path length is evaluated through the position vectors associated with the slave point at the beginning and the end of a time increment. Hence, quantities of the previous and current time steps will be denoted as ${ }^{n}(\bullet)$ and ${ }^{n+1}(\bullet)$, respectively. In the present study, the convective coordinates of the projection point in the last converged configuration ${ }^{n} \bar{\xi}=\left({ }^{n} \bar{\eta},{ }^{n} \bar{\zeta}\right)$ are used as the input parameters for the current time step. The variables from the last converged configuration ${ }^{n}(\bullet)$ are mapped forward to the current configuration using the notation ${ }^{n}(\tilde{\bullet})$. This means that these variables are evaluated in the current configuration using the convective coordinates from the previous time step, as illustrated in Fig. 4.

The simplest approximation for the slip path is given by the vector connecting the projection point calculated in the last converged configuration, mapped forward to the current configuration, and the solution point in the current configuration (see Fig. 4). It is expressed by:

$$
{ }^{n+1} \Delta \mathbf{g}={ }^{n+1} \overline{\mathbf{x}}^{\mathrm{m}}\left({ }^{n+1} \bar{\eta},{ }^{n+1} \bar{\zeta}\right)-{ }^{n} \tilde{\mathbf{x}}^{\mathrm{m}}\left({ }^{n} \bar{\eta},{ }^{n} \bar{\zeta}\right),
$$

where ${ }^{n+1} \overline{\mathbf{x}}^{\mathrm{m}}\left({ }^{n+1} \bar{\eta},{ }^{n+1} \bar{\zeta}\right)$ denotes the position vector of the projection point in the current configuration and ${ }^{n} \tilde{\mathbf{x}}^{\mathrm{m}}\left({ }^{n} \bar{\eta},{ }^{n} \bar{\zeta}\right)$ represents the position vector of the projection point in the last converged configuration, mapped into the current configuration. Since, in general, the slip vector (6) is not lying in the tangential plane of the contact surface (see Fig. 4), it is projected into the tangential plane defined by the surface normal vector at the solution point, evaluated in the current configuration. Thus, the tangential slip vector is given by:

$$
{ }^{n+1} \mathbf{g}_{\mathrm{t}}=\left(\mathbf{I}-{ }^{n+1} \overline{\mathbf{n}} \otimes{ }^{n+1} \overline{\mathbf{n}}\right)^{n+1} \Delta \mathbf{g}
$$

where ${ }^{n+1} \overline{\mathbf{n}}$ denotes the master surface normal vector at the solution point evaluated in the current time step, as illustrated in Fig. 4. Since the time step is typically very small in comparison with the curvature of the contact surface, the amplitude and direction of the tangential slip vector (7) is similar to slip path vector (6). The slip path length can be evaluated more accurately using curved paths [16,25,27], nevertheless Eq. (7) is adopted in this study due to its simplicity and because the direction of the tangential slip vector is more important than its length. In fact, the tangential 
slip vector defines the direction of the frictional force, while the slip path length is not directly used to evaluate the magnitude of the frictional force, which is based in the contact status (stick or slip) and the contact pressure.

The friction law defined at the contact interface establishes that the frictional force vector (tangential component of the Cauchy contact traction) is always collinear with the tangential slip vector, which is expressed by:

$$
\mathbf{g}_{\mathrm{t}}-\zeta \frac{\mathbf{t}_{\mathrm{t}}}{\left\|\mathbf{t}_{\mathrm{t}}\right\|}=\mathbf{0}
$$

where $\zeta$ is a consistency parameter. Note that the frictional force involved in (8) derives from the Cauchy contact traction defined in (4). The tangential slip vector expressed in (10) defines the tangential sliding of the slave point relatively to the master surface, as shown in Fig. 4. The frictional contact constraints expressed by means of the KKT conditions are given by:

$$
\Phi=\left\|\mathbf{t}_{\mathrm{t}}\right\|+\mu p_{\mathrm{n}} \leq 0 ; \quad \zeta \geq 0 ; \quad \Phi \zeta=0,
$$

where $\mu$ is the Coulomb friction coefficient. The first condition establishes the maximum magnitude for the frictional force, the second condition states that frictional force arises in the direction opposite to the relative motion (slip), while the last condition requires that such slip only occurs when $\left\|\mathbf{t}_{\mathrm{t}}\right\|=-\mu p_{\mathrm{n}}$. If the frictional force is less than the Coulomb limit $(\Phi<0)$, there is no motion between bodies in the tangential direction (stick contact status). These three conditions are usually denominated as friction law, slip rule and complementary condition, respectively. The Coulomb's cone is defined by the friction law, represented in the space of the normal and tangential traction components.

\subsection{Augmented Lagrangian method}

The virtual work principle for the two-body system can be defined as the sum of the virtual work of internal and external forces of the bodies and the virtual work of contact forces, which is classically written as:

$$
\delta W(\mathbf{u}, \delta \mathbf{u})=\delta W^{\text {int, ext }}(\mathbf{u}, \delta \mathbf{u})+\delta W^{\mathrm{c}}(\mathbf{u}, \delta \mathbf{u})=0,
$$

where $\mathbf{u}$ denotes the solution displacement field and $\delta \mathbf{u}$ represents the virtual displacements. The virtual work arising from the internal and external forces is denoted by $\delta W^{\text {int,ext }}$, while the contact contribution to the virtual work is defined as:

$$
\delta W^{\mathrm{c}}(\mathbf{u}, \delta \mathbf{u})=-\int_{\gamma_{\mathrm{c}}^{1}} \mathbf{t}^{1} \cdot \delta \mathbf{u}^{1} d \gamma_{\mathrm{c}}^{1}-\int_{\gamma_{\mathrm{c}}^{2}} \mathbf{t}^{2} \cdot \delta \mathbf{u}^{2} d \gamma_{\mathrm{c}}^{2} .
$$

Applying the balance of linear momentum across the contact interface $\mathbf{t}^{1} d \gamma_{\mathrm{c}}^{1}=-\mathbf{t}^{2} d \gamma_{\mathrm{c}}^{2}$, the contact virtual work can be represented as one integral over the master surface [53], defined by:

$$
\delta W^{\mathrm{c}}(\mathbf{u}, \delta \mathbf{u})=\int_{\gamma_{\mathrm{c}}^{2}} \mathbf{t}^{2} \cdot\left(\delta \mathbf{u}^{1}-\delta \mathbf{u}^{2}\right) d \gamma_{\mathrm{c}}^{2}=\int_{\gamma_{\mathrm{c}}^{2}}\left(p_{\mathrm{n}} \delta g_{\mathrm{n}}+\mathbf{t}_{\mathrm{t}} \cdot \delta \mathbf{g}_{\mathrm{t}}\right) d \gamma_{\mathrm{c}}^{2},
$$

where $\delta g_{\mathrm{n}}$ represents the variation of the normal gap function defined in (3) and $\delta \mathbf{g}_{\mathrm{t}}$ denotes variation of the tangential slip vector expressed in (7). The Cauchy contact traction is decomposed into normal and tangential components using (4).

In the present study, the augmented Lagrangian method is used to impose both the unilateral contact constraints (5) and the frictional contact constrains (9). The constrained minimization incremental problem is converted into a fully unconstrained problem [10,57]. Following Pietrzak and Curnier [20], the augmented Lagrangian functional is defined by:

$$
\mathcal{L}^{\mathrm{a}}\left(\mathbf{u}, \boldsymbol{\lambda}, \hat{p}_{\mathrm{n}}\right)=\Pi^{\mathrm{int}, \mathrm{ext}}(\mathbf{u})+\int_{\gamma_{\mathrm{c}}^{2}} l_{\mathrm{n}}\left(g_{\mathrm{n}}, \lambda_{\mathrm{n}}\right) d \gamma_{\mathrm{c}}^{2}+\int_{\gamma_{\mathrm{c}}^{2}} l_{\mathrm{t}}\left(\mathbf{g}_{\mathrm{t}}, \boldsymbol{\lambda}_{\mathrm{t}}, \hat{p}_{\mathrm{n}}\right) d \gamma_{\mathrm{c}}^{2},
$$

where $\Pi^{\mathrm{int}, \mathrm{ext}}(\mathbf{u})$ is a smooth potential energy of the system of contacting elastic bodies or its incremental homologue in plasticity (excluding the contact interactions). The closed forms for the augmented Lagrangian functionals $l_{\mathrm{n}}$ and $l_{\mathrm{t}}$ 
involved in (13) are given in the following:

$$
\begin{aligned}
& l_{\mathrm{n}}\left(g_{\mathrm{n}}, \lambda_{\mathrm{n}}\right)= \begin{cases}g_{\mathrm{n}} \hat{\lambda}_{\mathrm{n}}-\frac{\varepsilon}{2} g_{\mathrm{n}}^{2}, & \hat{\lambda}_{\mathrm{n}} \leq 0, \text { contact } \\
-\frac{1}{2 \varepsilon} \lambda_{\mathrm{n}}^{2}, & \hat{\lambda}_{\mathrm{n}}>0, \text { gap, }\end{cases} \\
& l_{\mathrm{t}}\left(\mathbf{g}_{\mathrm{t}}, \boldsymbol{\lambda}_{\mathrm{t}}, \hat{p}_{\mathrm{n}}\right)= \begin{cases}\hat{\boldsymbol{\lambda}}_{\mathrm{t}} \cdot \mathbf{g}_{\mathrm{t}}-\frac{\varepsilon}{2} \mathbf{g}_{\mathrm{t}} \cdot \mathbf{g}_{\mathrm{t}}, & \|-\mu \hat{p}_{\mathrm{n}}, \text { stick } \\
-\frac{1}{2 \varepsilon}\left(\boldsymbol{\lambda}_{\mathrm{t}} \cdot \boldsymbol{\lambda}_{\mathrm{t}}+2 \mu \hat{p}_{\mathrm{n}}\left\|\hat{\boldsymbol{\lambda}}_{\mathrm{t}}\right\|+\mu^{2} \hat{p}_{\mathrm{n}}^{2}\right), & \left\|\hat{\boldsymbol{\lambda}}_{\mathrm{t}}\right\|>-\mu \hat{p}_{\mathrm{n}}, \text { slip } \\
-\frac{1}{2 \varepsilon} \boldsymbol{\lambda}_{\mathrm{t}} \cdot \boldsymbol{\lambda}_{\mathrm{t}}, & \hat{p}_{\mathrm{n}}>0, \text { gap }\end{cases}
\end{aligned}
$$

where $\lambda_{\mathrm{n}}$ and $\lambda_{\mathrm{t}}$ are the Lagrange multipliers representing the contact pressure and the frictional force vector, respectively. Furthermore, the augmented Lagrange multipliers are denoted by a hat $\hat{\lambda}_{\mathrm{n}}=\lambda_{\mathrm{n}}+\varepsilon g_{\mathrm{n}}$ and $\hat{\boldsymbol{\lambda}}_{\mathrm{t}}=\boldsymbol{\lambda}_{\mathrm{t}}+\varepsilon \mathbf{g}_{\mathrm{t}}$, where $\varepsilon$ denotes the non-negative penalty parameter. Due to the non-associated character of the Coulomb friction law, $\hat{p}_{\mathrm{n}}$ is a regularized contact pressure at the solution, which defines the radius of the Coulomb's cone. Note that the tangential regularized functional $l_{\mathrm{t}}$ is defined also for the non-contact domain $\hat{p}_{\mathrm{n}}>0$.

Since the augmented Lagrangian functional (13) is $C^{1}$ continuous [20,58], the frictional contact problem can be reformulated as the following unconstrained saddle-point problem:

$$
\min _{\mathbf{u}} \max _{\lambda} \mathcal{L}^{\mathrm{a}}\left(\mathbf{u}, \boldsymbol{\lambda}, \hat{p}_{\mathrm{n}}\right)
$$

where the solution minimizes the functional by $\mathbf{u}$ and maximizes by $\boldsymbol{\lambda}$. However, this saddle-point problem is not a standard min-max problem since the frictional force depends on the normal contact pressure, which is a part of the solution. In order to obtain the augmented Lagrangian frictional contact virtual work, the saddle-point stationary condition is expressed by $\delta \mathcal{L}^{\mathrm{a}}\left(\mathbf{u}, \boldsymbol{\lambda}, \hat{p}_{\mathrm{n}}\right)=0$, obtaining the following expression:

$$
\delta W^{\text {int,ext }}(\mathbf{u}, \delta \mathbf{u})+\int_{\gamma_{\mathrm{c}}^{2}}\left(\frac{\partial l_{\mathrm{n}}}{\partial g_{\mathrm{n}}} \delta g_{\mathrm{n}}+\frac{\partial l_{\mathrm{t}}}{\partial \mathbf{g}_{\mathrm{t}}} \cdot \delta \mathbf{g}_{\mathrm{t}}\right) d \gamma_{\mathrm{c}}^{2}+\int_{\gamma_{\mathrm{c}}^{2}}\left(\frac{\partial l_{\mathrm{n}}}{\partial \lambda_{\mathrm{n}}} \delta \lambda_{\mathrm{n}}+\frac{\partial l_{\mathrm{t}}}{\partial \boldsymbol{\lambda}_{\mathrm{t}}} \cdot \delta \boldsymbol{\lambda}_{\mathrm{t}}\right) d \gamma_{\mathrm{c}}^{2}=0,
$$

where the variation of the potential energy $\Pi^{\text {int,ext }}(\mathbf{u})$ yields the virtual work arising from the internal and external forces $\delta \Pi^{\mathrm{int}, \mathrm{ext}}=\delta W^{\mathrm{int}, \mathrm{ext}}(\mathbf{u}, \delta \mathbf{u})$. The derivatives of the functionals $l_{\mathrm{n}}$ and $l_{\mathrm{t}}$ involved in the augmented virtual work developed by contact and friction forces are given as follows, for the three possible contact statuses of a slave point (gap, stick and slip):

$$
\begin{aligned}
& \frac{\partial l_{\mathrm{n}}\left(g_{\mathrm{n}}, \lambda_{\mathrm{n}}\right)}{\partial g_{\mathrm{n}}}= \begin{cases}\hat{\lambda}_{\mathrm{n}}, & \hat{\lambda}_{\mathrm{n}} \leq 0, \text { contact } \\
0, & \hat{\lambda}_{\mathrm{n}}>0, \text { gap, }\end{cases} \\
& \frac{\partial l_{\mathrm{n}}\left(g_{\mathrm{n}}, \lambda_{\mathrm{n}}\right)}{\partial \lambda_{\mathrm{n}}}= \begin{cases}g_{\mathrm{n}}, & \hat{\lambda}_{\mathrm{n}} \leq 0, \text { contact } \\
-\frac{\lambda_{\mathrm{n}}}{\varepsilon}, & \hat{\lambda}_{\mathrm{n}}>0, \text { gap, }\end{cases} \\
& \frac{\partial l_{\mathrm{t}}\left(\mathbf{g}_{\mathrm{t}}, \boldsymbol{\lambda}_{\mathrm{t}}\right)}{\partial \mathbf{g}_{\mathrm{t}}}= \begin{cases}\hat{\lambda}_{\mathrm{t}}, & \left\|\hat{\lambda}_{\mathrm{t}}\right\| \leq-\mu \hat{p}_{\mathrm{n}}, \text { stick } \\
-\mu \hat{p}_{\mathrm{n}} \frac{\hat{\lambda}_{\mathrm{t}}}{\left\|\hat{\lambda}_{\mathrm{t}}\right\|}, & \left\|\hat{\boldsymbol{\lambda}}_{\mathrm{t}}\right\|>-\mu \hat{p}_{\mathrm{n}}, \text { slip } \\
0, & \left\|\hat{\boldsymbol{\lambda}}_{\mathrm{t}}\right\| \leq-\mu \hat{p}_{\mathrm{n}}, \text { stick }\end{cases} \\
& \frac{\partial l_{\mathrm{t}}\left(\mathbf{g}_{\mathrm{t}}, \boldsymbol{\lambda}_{\mathrm{t}}\right)}{\partial \boldsymbol{\lambda}_{\mathrm{t}}}= \begin{cases}\mathbf{g}_{\mathrm{t}}, & \hat{p}_{\mathrm{n}}>0, \text { gap, } \\
-\frac{1}{\varepsilon_{\mathrm{t}}}\left(\lambda_{\mathrm{t}}+\mu \hat{p}_{\mathrm{n}} \frac{\hat{\lambda}_{\mathrm{t}}}{\left\|\hat{\lambda}_{\mathrm{t}}\right\|}\right), & \left\|\hat{\lambda}_{\mathrm{t}}\right\|>-\mu \hat{p}_{\mathrm{n}}, \text { slip } \\
-\frac{1}{\varepsilon_{\mathrm{t}}} \lambda_{\mathrm{t}}, & \hat{p}_{\mathrm{n}}>0, \text { gap. }\end{cases}
\end{aligned}
$$


In the finite element method framework, the augmented Lagrangian virtual work principle and the Lagrange multiplier equations represent a set of nonlinear equations for primal (displacements) and dual (contact forces) variables. These equations are solved using the generalized Newton method [10,59]. Note that the regularized contact pressure $\hat{p}_{\mathrm{n}}$, which describes the augmented radius of Coulomb's disk (section of the Coulomb's cone), becomes an unknown and is replaced by the augmented Lagrangian multiplier $\hat{\lambda}_{n}$. The linearization of the contact virtual work principle is also performed with respect to this variable [20].

\section{Surface smoothing method}

The main idea behind the proposed 3D surface smoothing procedure is to combine the accuracy achieved using the Nagata interpolation [49,52] with the efficiency of classical linear finite elements. Hence, the contact surface is composed by individual Nagata patches associated with each facet, while the bulk is discretized with linear elements. This ensures a more accurate evaluation of the kinematic contact variables (normal gap function and tangential slip vector), as well as the elimination (or at least reduction) of the discontinuity in the contact surface normal vector. Thus, several drawbacks associated with the classical piecewise bilinear representation of the master surface are eliminated by adopting this contact surface smoothing method.

Each bilinear facet composing the master contact surface is replaced by a Nagata patch, which is defined only by the nodes of the facet and the normal vector in each node. This nodal normal vectors are approximated using the weighted average of the normal vectors of all facets adjacent to the master node [60], as explained in detail in Section 3.3.

\subsection{Nagata patch interpolation}

The Nagata patch interpolation was developed by Nagata [49] for interpolating discretized surfaces in order to recover the original geometry with good accuracy. Its central idea is the quadratic interpolation, requiring only the position and normal vectors at the nodes of the surface mesh. The local support of the adopted interpolation method allows to handle irregular surface finite element meshes, as well as hybrid surface meshes (see Fig. 1(c)). Moreover, the low order interpolation degree (quadratic) and its simplicity allows to obtain a computationally attractive approach. On the other hand, it only achieves $G^{1}$ continuity (direction of the tangent vector is continuous) between patches at the nodes [52]. The accuracy of the surface smoothing procedure is evaluated in Section 3.2.

Considering the simplest case of a $2 \mathrm{D}$ interpolation, an edge defined by its end points with position vectors $\mathbf{x}_{0}$ and $\mathbf{x}_{1}$ gives a Nagata curve in the form:

$$
\mathcal{C}(\xi)=\mathbf{x}_{0}+\left(\mathbf{x}_{1}-\mathbf{x}_{0}-\mathbf{c}\right) \xi+\mathbf{c} \xi^{2},
$$

where $\xi$ is the local coordinate that satisfies the condition $0 \leq \xi \leq 1$. The coefficient vector $\mathbf{c}$, called the curvature parameter, adds the curvature to the edge. Requiring that the Nagata curve (22) is orthogonal to the unit normal vectors $\mathbf{n}_{0}$ and $\mathbf{n}_{1}$ defined at the nodes, as shown in Fig. 5(a), the curvature parameter is given as follows:

$$
\mathbf{c}\left(\mathbf{x}_{0}, \mathbf{x}_{1}, \mathbf{n}_{0}, \mathbf{n}_{1}\right)= \begin{cases}\frac{\left[\mathbf{n}_{0}, \mathbf{n}_{1}\right]}{1-a^{2}}\left[\begin{array}{cc}
1 & -a \\
-a & 1
\end{array}\right]\left\{\begin{array}{c}
\mathbf{n}_{0} \cdot\left(\mathbf{x}_{1}-\mathbf{x}_{0}\right) \\
-\mathbf{n}_{1} \cdot\left(\mathbf{x}_{1}-\mathbf{x}_{0}\right)
\end{array}\right\} & (a \neq \pm 1) \\
\frac{\left[\mathbf{n}_{0}, \pm \mathbf{n}_{0}\right]}{2}\left\{\begin{array}{c}
\mathbf{n}_{0} \cdot\left(\mathbf{x}_{1}-\mathbf{x}_{0}\right) \\
\mp \mathbf{n}_{0} \cdot\left(\mathbf{x}_{1}-\mathbf{x}_{0}\right)
\end{array}\right\}=\mathbf{0} & (a= \pm 1),\end{cases}
$$

where $a=\mathbf{n}_{0} \cdot \mathbf{n}_{1}$ denotes the cosine of the angle between the two normal vectors. When the normal vectors are parallel $(a= \pm 1)$, the curvature parameter vanishes and the Nagata curve degenerates into a straight segment. The interpolation of an edge is the basis to apply the Nagata interpolation to general $n$-sided patches, such as triangular and quadrilateral patches (see Fig. 5). First, each edge composing the bilinear facet is interpolated through the quadratic curve (22) and then the interior of the Nagata patch is filled by its trace [49].

In case of a quadrilateral Nagata patch, schematically presented in Fig. 5(c), it is given by the following quadratic polynomial:

$$
\mathcal{P}_{\mathrm{q}}(\eta, \zeta)=\mathbf{c}_{00}+\mathbf{c}_{10} \eta+\mathbf{c}_{01} \zeta+\mathbf{c}_{11} \eta \zeta+\mathbf{c}_{20} \eta^{2}+\mathbf{c}_{02} \zeta^{2}+\mathbf{c}_{21} \eta^{2} \zeta+\mathbf{c}_{12} \eta \zeta^{2},
$$

where $\eta$ and $\zeta$ are the local coordinates satisfying the patch domain validity expressed by $0 \leq \eta, \zeta \leq 1$. The eight coefficient vectors $\mathbf{c}_{i j}$ are calculated using only the position and surface normal vectors at the master nodes, which are 
a

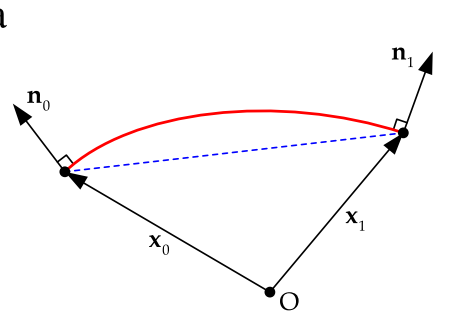

b

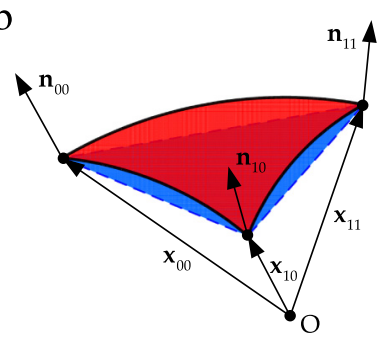

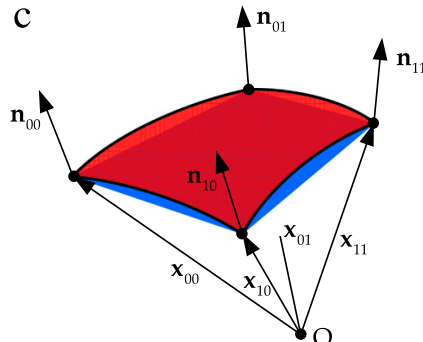

Fig. 5. Nagata patch interpolation: (a) curve; (b) triangular patch; (c) quadrilateral patch.

defined as follows:

$$
\begin{aligned}
& \mathbf{c}_{00}=\mathbf{x}_{00}, \\
& \mathbf{c}_{10}=\mathbf{x}_{10}-\mathbf{x}_{00}-\mathbf{c}_{1}, \\
& \mathbf{c}_{01}=\mathbf{x}_{01}-\mathbf{x}_{00}-\mathbf{c}_{4}, \\
& \mathbf{c}_{11}=\mathbf{x}_{11}-\mathbf{x}_{10}-\mathbf{x}_{01}+\mathbf{x}_{00}+\mathbf{c}_{1}-\mathbf{c}_{2}-\mathbf{c}_{3}+\mathbf{c}_{4}, \\
& \mathbf{c}_{20}=\mathbf{c}_{1}, \\
& \mathbf{c}_{02}=\mathbf{c}_{4}, \\
& \mathbf{c}_{21}=\mathbf{c}_{3}-\mathbf{c}_{1}, \\
& \mathbf{c}_{12}=\mathbf{c}_{2}-\mathbf{c}_{4},
\end{aligned}
$$

where $\mathbf{c}_{1}, \mathbf{c}_{2}, \mathbf{c}_{3}$ and $\mathbf{c}_{4}$ are the coefficient vectors defined by (23) for the edges $\left(\mathbf{x}_{00}, \mathbf{x}_{10}\right),\left(\mathbf{x}_{10}, \mathbf{x}_{11}\right),\left(\mathbf{x}_{01}, \mathbf{x}_{11}\right)$ and $\left(\mathbf{x}_{00}, \mathbf{x}_{01}\right)$, respectively. The triangular Nagata patch, schematically presented in Fig. 5(b), is obtained in a similar way as the quadrilateral patch [61].

In opposition to the smoothing procedures based on least-squares approximations with polynomial basis [28,29,62], the evaluation of the Nagata patch interpolation coefficients expressed in (25) does not requires solving any system of equations (matrix inversion). In fact, the interpolation coefficients are calculated via the closed form of the curvature parameter given in (23). However, the quadratic degree of the Nagata interpolation does not allow to generate curves with inflection. Indeed, very sharp patches with inverted orientation can arise for specific arrangements of the nodal normal vectors directions [63]. Therefore, since the surface normal vector orientation defines the sign of the normal gap function (3), the contact status (gap or contact) can be wrongly estimated in some situations. In order to avoid such problems, some modifications in the curvature parameter (23) were proposed by Neto et al. [61], which are based in geometrical considerations. In fact, additional constraints are introduced to prevent the flapping of the patches, replacing the quadratic interpolation by linear interpolation.

\subsection{Accuracy in the contact surface representation}

The surface smoothing procedure intends to improve the accuracy of the contact surface representation, allowing a more accurate evaluation of the kinematic contact variables. The comparison between the classical piecewise bilinear representation and the surface smoothing method based in the Nagata patch interpolation is presented in this section. The circular arc (2D) and the sphere (3D) are the geometries selected to perform this analysis due to their wide application in surface modelling. The nodal normal vectors required for the Nagata interpolation are obtained from the analytical functions. The accuracy achieved in the contact surface representation is evaluated by means of two distinct types of error: radial error and surface normal vector error [15,50]. Considering a sphere of radius $r$, the radial error in the surface interpolation is defined by:

$$
\delta_{\mathrm{r}}(\eta, \zeta)=\frac{(\mathcal{P}(\eta, \zeta)-\mathbf{o}) \cdot \mathbf{n}_{\text {analy }}-r}{r},
$$

where $\mathcal{P}(\eta, \zeta)$ denotes the position vector of a generic point on the interpolated surface, $\mathbf{o}$ is the position vector of the sphere centre and $\mathbf{n}_{\text {analy }}$ represents the unit normal vector of the spherical surface, given by the analytic function. 

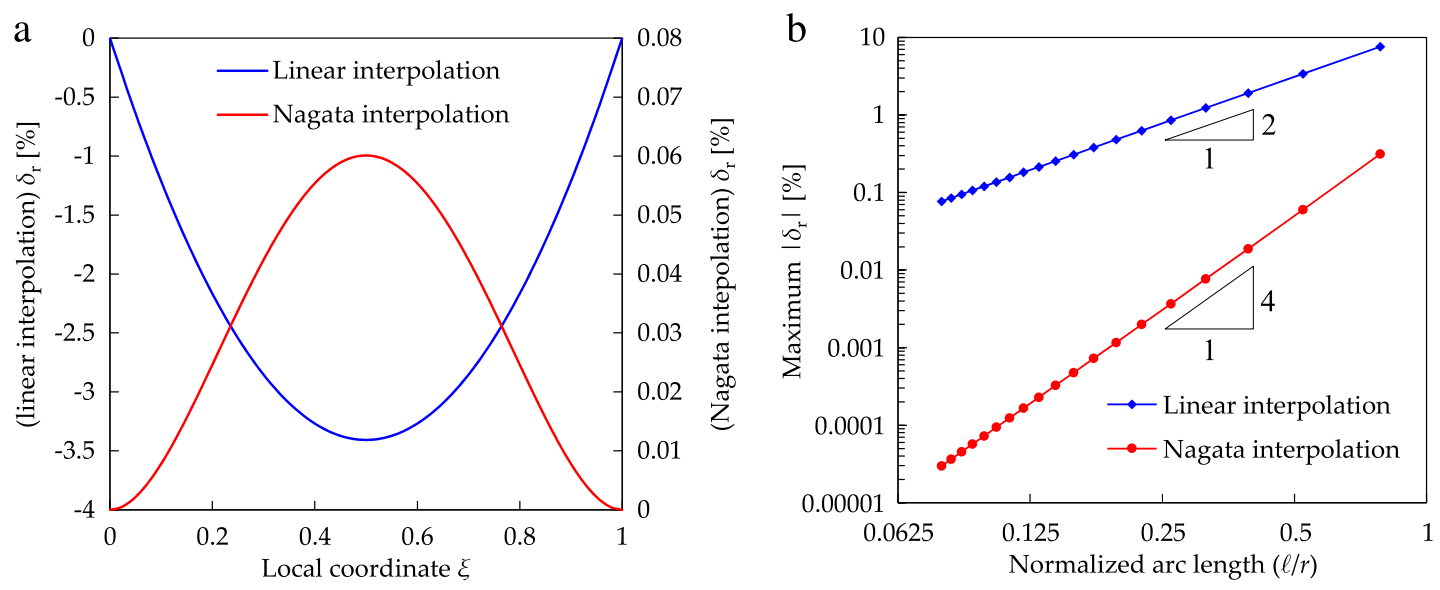

Fig. 6. Radial error in a circular arc using linear and Nagata interpolation: (a) distribution for an arc with central angle of $30^{\circ}$; (b) maximum value of the modulus as function of the normalized arc length.

This error indicates the dimensionless distance (measured in the radial direction) between the discretized surface and the analytical sphere. It is directly related with the accuracy achieved in the computation of the normal gap function (3). The other error studied is the surface normal vector error, which is defined in terms of modulus by:

$$
\left|\delta_{\mathrm{n}}(\eta, \zeta)\right|=\cos ^{-1}\left(\mathbf{n}_{\text {Nagata }}(\eta, \zeta) \cdot \mathbf{n}_{\text {analy }}\right),
$$

where $\mathbf{n}_{\text {Nagata }}$ is the unit normal vector of the interpolated surface, which is defined by the cross product of the two partial derivatives. The modulus of the normal vector error expresses the angle between the normal vector of the interpolated surface and the analytical normal vector. This error is connected with the non-physical oscillations in the contact force for large sliding contact problems, which are induced by the orientation of the normal to the contact surface.

The Nagata interpolation allows to create patches with or without recovering their curvature. Thus, in this study, the piecewise bilinear representation of the surfaces is defined through the Nagata patch interpolation setting to zero the curvature parameter defined in (23). The comparison between linear and Nagata interpolation in terms of radial error is presented in Fig. 6 for the circular arc. The radial error distribution in a circular arc with a central angle of $30^{\circ}$, described by a single curve, is shown in Fig. 6(a). The maximum value of error is located at the middle of the curve for both interpolation methods, which is approximately $-3.5 \%$ (inside the circular arc) in the linear interpolation and $0.06 \%$ (outside the circular arc) in the Nagata interpolation. Since the order of magnitude in the results is not comparable, the figure presents two different scales. The evolution of the maximum error value (modulus) as a function of the normalized arc length $(\ell / r)$, i.e. the mesh refinement, is presented in Fig. $6(\mathrm{~b})$. The range considered for the normalized arc length is from 0.0785 until 0.157 , which corresponds to dividing a quarter of circle from 2 to 20 equal segments, respectively. The maximum value of error decreases quadratically with the normalized arc length when adopting linear interpolation, while when applying Nagata interpolation the convergence rate is quartic $[49,61]$.

The error in the normal vector orientation is presented in Fig. 7, comparing linear and Nagata interpolations applied to the circular arc. The error distribution in a single curve describing an arc with $30^{\circ}$ of central angle is shown in Fig. 7(a). The discontinuity of the normal vector between adjacent linear finite elements is highlighted through the error value at the nodes, which is non-zero and presents opposite signals. On the other hand, the Nagata interpolation assures the $G^{1}$ continuity across curves due to the imposed nodal normal vector. The maximum value of the normal vector error in the linear interpolation is $15^{\circ}$ (half value of the arc central angle), while in the Nagata interpolation it is only $0.2^{\circ}$. The evolution of the maximum error (modulus) as a function of the normalized arc length $(\ell / r)$ is presented in Fig. 7(b). The maximum value decreases linearly when the linear interpolation is adopted, while the Nagata interpolation method provides a cubic convergence rate $[49,61]$.

The spherical surface discretized by traditional piecewise bilinear finite elements and smoothed with Nagata patches is presented in Fig. 8(a) and (b), respectively. The radial error is negative in the faceted surface description, either using triangular or quadrilateral finite elements. On the other hand, the Nagata interpolation leads to a surface 

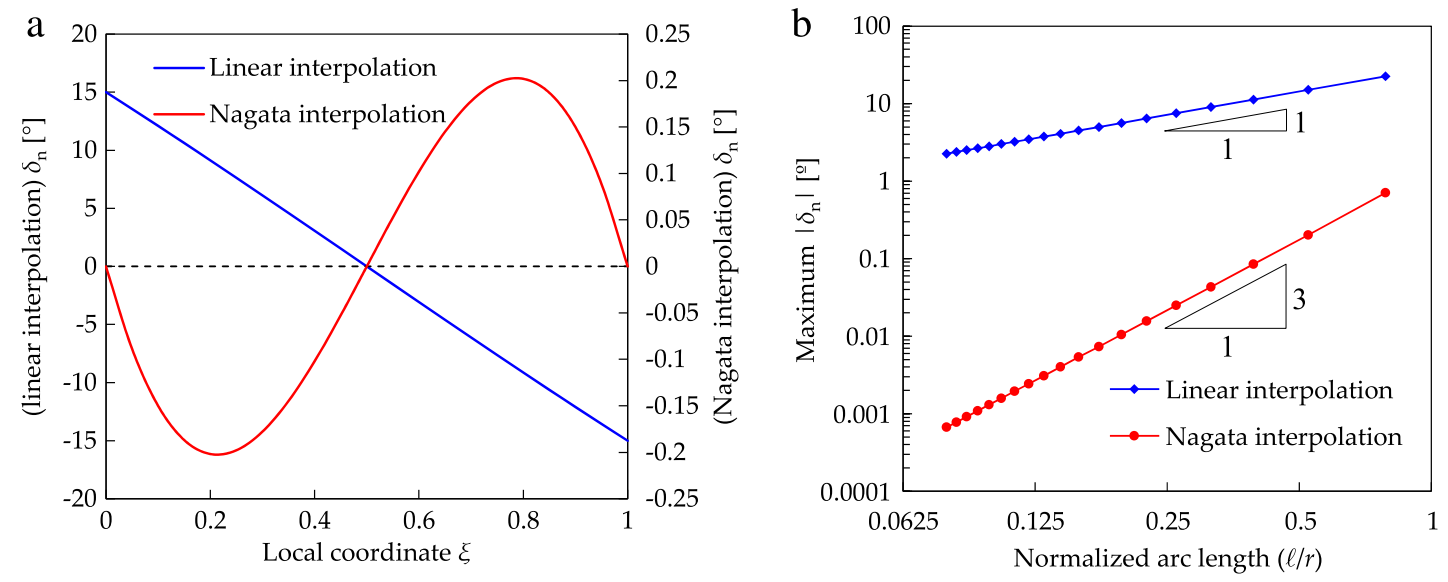

Fig. 7. Normal vector error in a circular arc using linear and Nagata interpolation: (a) distribution for an arc with central angle of $30^{\circ}$; (b) maximum value of the modulus as function of the normalized arc length.
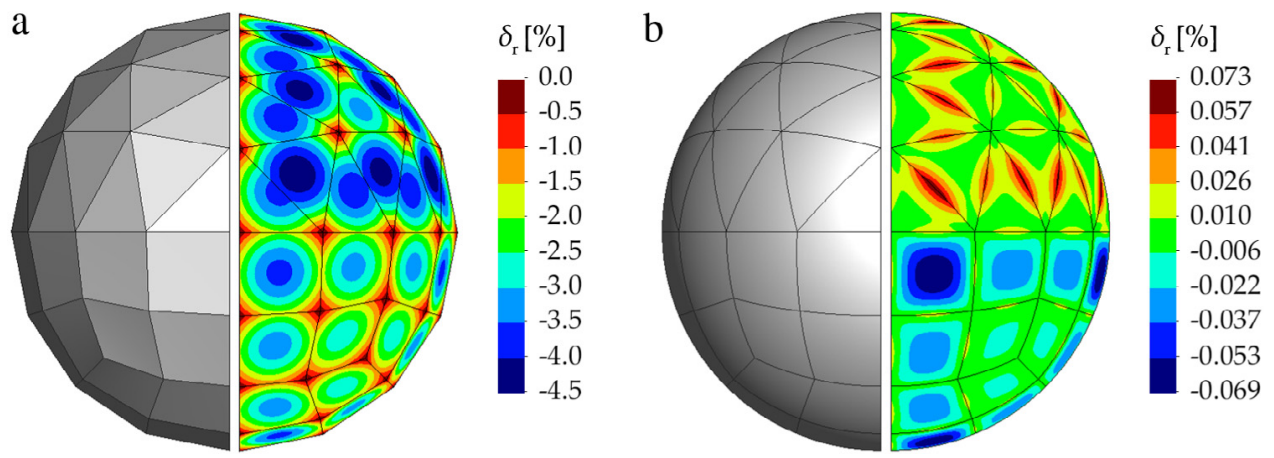

Fig. 8. Radial error distribution in the spherical surface described by: (a) bilinear finite elements; (b) Nagata patches.

with radial error predominantly positive in the triangular patches and negative in the quadrilateral patches, as shown in Fig. 8(b). The range of the radial error decreases from $4.5 \%$ to only $0.14 \%$ when the Nagata interpolation is applied (see Fig. 8). In fact, the maximum value of the radial error in the smoothed surface decreases quartically with the square root of the element area normalized by the sphere radius, as showed in [52]. The distribution of the surface normal vector error for both surface description methods is presented in Fig. 9. Considering the piecewise bilinear finite element representation of the spherical surface, the maximum value of error arises in the nodes (see Fig. 9(a)), which is approximately $17^{\circ}$. On the other hand, when applying the Nagata patch interpolation in the surface smoothing, the maximum value of error occurs in the edges middle, as shown in Fig. 9(b), and it is significantly inferior (lower than $1^{\circ}$ ). For both surface description methods, the normal vector angle error decreases with the mesh refinement. The faceted surface description exhibits a linear convergence rate, while the Nagata smoothing method provides a cubic order of convergence [52]. Although the smoothing method with Nagata patches does not guarantee $G^{1}$ continuity at the boundaries between patches, the low value of error in the surface normal vector (see Fig. 9(b)) and the fast convergence rate with the mesh refinement allows to assume quasi- $G^{1}$ continuity.

\subsection{Nodal normal vector approximation}

The Nagata patch interpolation requires the knowledge of the surface normal vector in each node of the surface mesh, as highlighted in the definition of the curvature parameter (23). Nevertheless, the finite element mesh of the master surface only comprises the coordinates of the master nodes and the finite element connectivity. In the particular case of rigid contact surfaces, the finite element mesh is usually generated from a CAD model, allowing the use the information contained herein (e.g. IGES file format) to evaluate the nodal normal vectors [64]. However, in the general case of contact between deformable bodies, the nodal normal vectors must be estimated using the information 

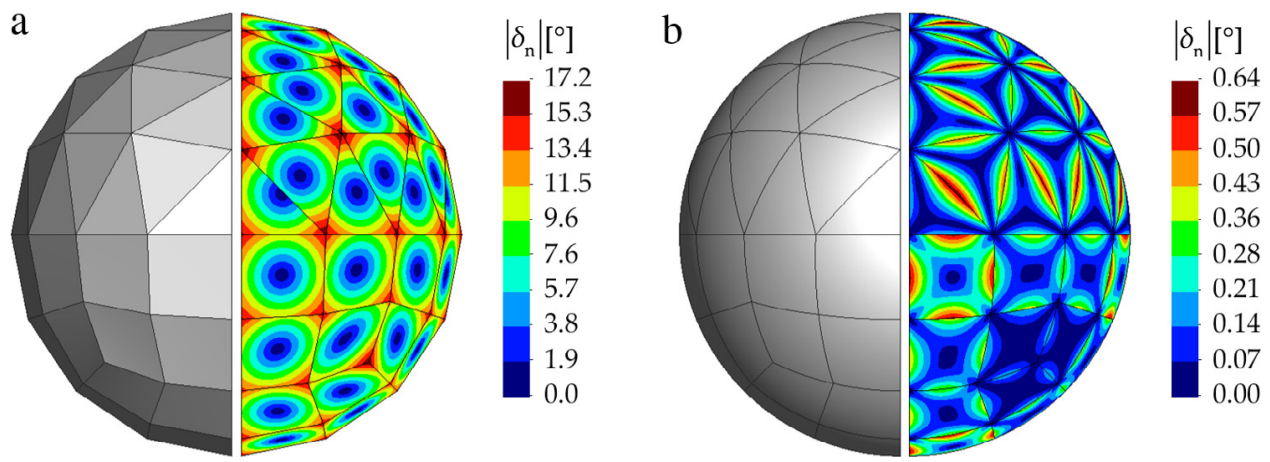

Fig. 9. Normal vector error distribution in the spherical surface described by: (a) bilinear finite elements; (b) Nagata patches.
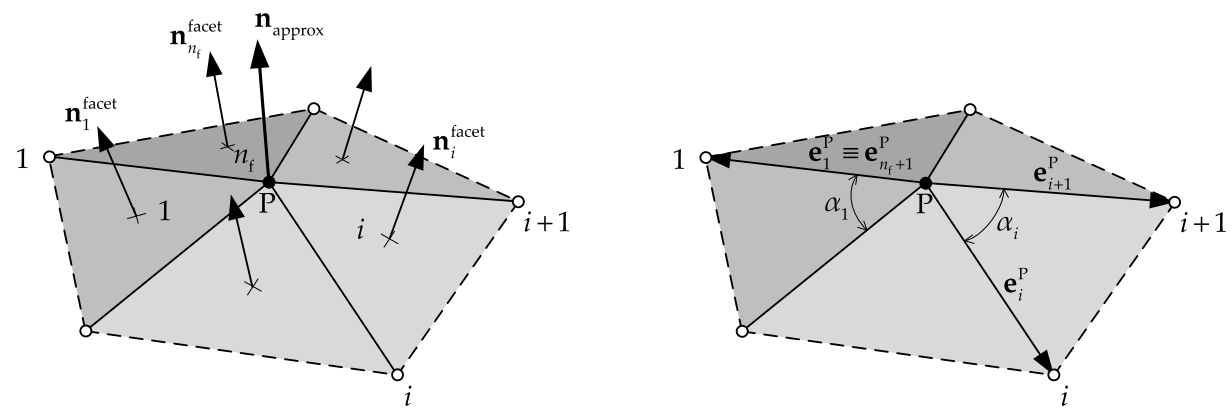

Fig. 10. Schematic representation of the nodal normal vector approximation using the normal vectors of the surrounding facets, including the notation adopted.

about the neighbouring finite elements [65]. In the present study, the normal vector required in each master node is approximated using the weighted average of the normal vectors of all facets adjacent to the master node, following the approach presented by Jin et al. [60].

The unit normal vector of each bilinear facet shared by a master node is defined by the cross product of its two reciprocal edges $\mathbf{n}_{i}^{\text {facet }}=\mathbf{e}_{i}^{\mathrm{P}} \times \mathbf{e}_{i+1}^{\mathrm{P}} /\left\|\mathbf{e}_{i}^{\mathrm{P}} \times \mathbf{e}_{i+1}^{\mathrm{P}}\right\|$, as illustrated in Fig. 10. Then, the surface normal vector in the master node is obtained from the weighted sum of the normal vectors of the neighbouring facets (finite elements). The approximated unit normal vector at a generic master node of the surface finite element mesh, surrounded by $n_{\mathrm{f}}$ facets, is expressed by:

$$
\mathbf{n}_{\text {approx }}=\sum_{i=1}^{n_{\mathrm{f}}} w_{i} \mathbf{n}_{i}^{\text {facet }} /\left\|\sum_{i=1}^{n_{\mathrm{f}}} w_{i} \mathbf{n}_{i}^{\text {facet }}\right\|,
$$

where $w_{i}$ denotes the weight associated with the $i$ th finite element (facet) surrounding the master node. The graphical representation of (28) is illustrated in Fig. 10 for a master node surrounded by 5 triangular finite elements. When quadrilateral finite elements (generally non-coplanar) are adopted in the contact surface description (see Fig. 1(b)), the normal vector of each facet required for (28) is evaluated using the two reciprocal edges that share the node.

Several weighting factors have been developed taking into account different surface properties [60]. The simplest was introduced by Gouraud [66], which will be referred as the mean weighted equally (MWE), since it provides the same weight for all facets:

$$
w_{i}^{\mathrm{MWE}}=1,
$$

where each adjacent facet contributes equally to the nodal normal vector approximation. The second weighting factor was proposed by Thürmer and Wüthrich [67], which uses the incident angle of each facet as weight. Defining the angle between the two edges of the $i$ th facet by $\alpha_{i}$ (see Fig. 10), the weighting factor of each facet is expressed as:

$$
w_{i}^{\mathrm{MWA}}=\alpha_{i},
$$


which will be referred as the mean weighted by angle (MWA). The next two weighting factors were developed by Max [68]. The first one, referred as the mean weighted by areas of adjacent triangles (MWAAT), defines the weighting factor by the area of the triangle formed by the two edges incident on the node:

$$
w_{i}^{\text {MWAAT }}=\left\|\mathbf{e}_{i}^{\mathrm{P}}\right\|\left\|\mathbf{e}_{i+1}^{\mathrm{P}}\right\| \sin \left(\alpha_{i}\right)=\left\|\mathbf{e}_{i}^{\mathrm{P}} \times \mathbf{e}_{i+1}^{\mathrm{P}}\right\|,
$$

where $\mathbf{e}_{i}^{\mathrm{P}}$ and $\mathbf{e}_{i+1}^{\mathrm{P}}$ denote the vectors representing the edges incident on the master node, as schematically illustrated in Fig. 10. The other weighting factor proposed by Max [68] is referred as the mean weighted by sine and edge length reciprocals (MWSELR), which is expressed for each facet by:

$$
w_{i}^{\mathrm{MWSELR}}=\frac{\sin \left(\alpha_{i}\right)}{\left\|\mathbf{e}_{i}^{\mathrm{P}}\right\|\left\|\mathbf{e}_{i+1}^{\mathrm{P}}\right\|},
$$

which takes into account the differences in size of the adjacent edges, as well as the angle between them. Note that this weighting factor was derived considering that the surface fitting the nodes is spherical. Thus, it provides the exact normal vector if the discretized surface is a sphere.

In order to assess the accuracy of each weighting factor involved in the approximation of the nodal normal vector, three different regular finite element meshes of a spherical surface are adopted, which are similar to the one shown in Fig. 8. The coarse mesh is composed by 448 finite elements, the medium mesh involves 1372 finite elements, while the fine mesh comprises 2800 finite elements. The error in the nodal normal vector approximation is defined by the following expression:

$$
\theta=\cos ^{-1}\left(\mathbf{n}_{\text {approx }} \cdot \mathbf{n}_{\text {analy }}\right),
$$

where $\mathbf{n}_{\text {approx }}$ is the approximated nodal normal vector given in (28) and $\mathbf{n}_{\text {analy }}$ denotes the unit normal vector evaluated from the analytical function. This error is evaluated in each node of the surface mesh and it represents the angle between the analytical and the approximated normal vectors.

The cumulative frequency histogram of the angular error in the nodal normal vector approximation for the spherical surface is presented in Fig. 11. The accuracy of the weighting factors defined in (29)-(31) is compared for the three finite element meshes. Note that the weighting factor expressed by (32) is not studied since it provides the exact normal vector in case of spherical surfaces. The surface mesh refinement reduces both the maximum and the median value of the error in the nodal normal vector approximation, as shown in Fig. 11. The maximum discrepancy between the approximated and the analytical nodal normal vector arises in the transition between triangular and quadrilateral finite elements, which is approximately $1.1^{\circ}$ in the coarse mesh using the MWA weighting factor. In all meshes, the normal vectors obtained with the MWAAT weighting factor are worse than the others. Nevertheless, it is used by Puso and Laursen [26] to define the nodal normal vectors required for Gregory patch interpolation applied in the contact smoothing method. Considering the example of a spherical surface, the MWA weighting factor provides the best approximation, resulting in a median value of approximately $0.11^{\circ}, 0.03^{\circ}$ and $0.02^{\circ}$ for the coarse, medium and fine meshes, respectively (see Fig. 11). Nevertheless, the weighting factor given in (32) is adopted in all numerical examples of Section 5, since it provides the most accurate overall results [61].

The approximated nodal normal vector provided by the weighted average (28) is modified in the nodes located in symmetry planes, in order to provide a normal vector laying on the symmetry plane. A similar approach is also applied in the transition between flat and curved surfaces [61], which is based in the comparison between the normal vector of each facet and the approximated nodal normal vector. The influence of the approximated nodal normal vectors in the Nagata interpolation accuracy was recently studied by Neto et al. [64]. They concluded that the interpolation error is slightly affected for small values of error in the nodal normal vector. Indeed, the shape error can decreases in specific 2D situations.

\section{Node-to-Nagata contact elements}

The discretization of the contact interface is performed in the present study with the node-to-segment (NTS) approach, which is commonly used to solve large deformation and large sliding contact problems [41]. The impenetrability and friction constraints are enforced at the slave nodes, which are checked for contact with the 


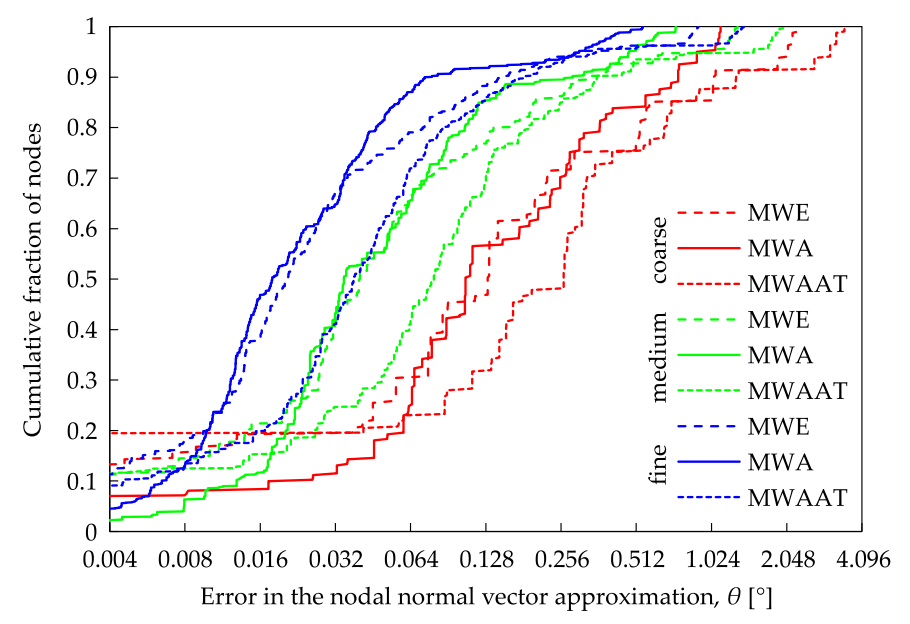

Fig. 11. Cumulative frequency histogram of the angular error in the nodal normal vector approximation for three distinct finite element meshes of a spherical surface.

discretized master surface defined by segments (or facets) [59]. In this work, the master surface is discretized either by triangular (based on the 4-node tetrahedral finite element) or quadrilateral (based on the 8-node hexahedral finite element) bilinear finite elements. Then, the proposed surface smoothing method (see Section 3) is applied in order to obtain a master surface described by Nagata patches. Each contact element is defined by one slave node, a Nagata patch (defined with three or four master nodes) and an artificial node containing the contact force (Lagrange multipliers) as degrees of freedom. The connection between the contacting bodies is performed through the contact elements, transferring the contact efforts from the slave to the master surface according to the impenetrability and friction conditions.

\subsection{Contact detection procedure}

The contact detection is the step preceding the creation of the contact elements, which aims to determine the contacting pairs, i.e. define for each slave node the corresponding master Nagata patch. It is typically decomposed into two phases: global search and local search [2]. The global contact search procedure adopted in the present work is based on selecting the closest master node for each slave node, as proposed by Benson and Hallquist [69]. Afterwards, all Nagata patches having the master node as one of their vertices are selected for the local contact search. Due to the large sliding and finite deformation of the bodies, the global search procedure is performed in each increment (i.e. the set of Nagata patches candidate to establish contact with each slave node is updated). The local contact search procedure evaluates the local coordinates of the closest point (cf. Fig. 3) that minimizes the normal gap function [4]. The closest point projection is the key feature of the local search procedure since it dictates the value of the kinematic contact variables (see Section 2.1), for each slave node.

Assuming that the Nagata patch is expressed by $\mathcal{P}(\eta, \zeta)$ and the position vector of the slave node is denoted by $\mathbf{x}^{\mathrm{s}}$, the closest point projection consists in finding the local coordinates and the normal gap function, such that:

$$
\mathcal{P}(\eta, \zeta)+g_{\mathrm{n}} \mathbf{n}(\eta, \zeta)-\mathbf{x}^{\mathrm{s}}=\mathbf{0},
$$

where $\mathbf{n}(\eta, \zeta)$ is the unit normal vector of the Nagata patch. The nonlinear system of equation (34) is solved numerically using the Newton-Raphson method, providing simultaneously the normal gap value and the local coordinates of the contact point on the master surface [31]. The midpoint of the patch is the initial guess for the iterative procedure. The required Jacobian matrix comprises the partial derivatives of the Nagata patch and the gradient of the normal vector with respect to the local coordinates, which can be calculated using the Weingarten formula $[31,70]$.

The surface smoothing approach allows to improve the contact surface representation and provides a continuous projection of the slave nodes on the discretized master surface. In fact, the classical piecewise bilinear finite element representation of the master surface leads to numerical difficulties in the evaluation of the normal gap function (3) and the tangential slip vector (7), which are strongly connected with the closest point projection algorithm. Each master 
a

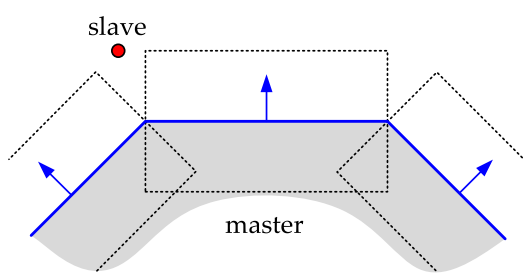

b

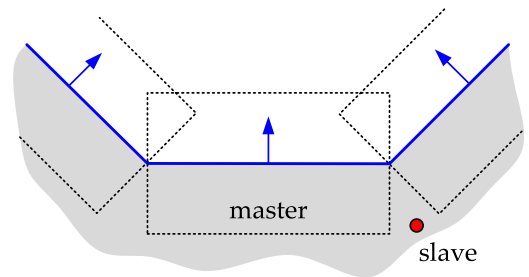

Fig. 12. Difficulties associated with the closest point projection considering the faceted description of the master surface: (a) slave node near a sharp corner in a convex surface; (b) slave node near a valley in a concave surface.

a

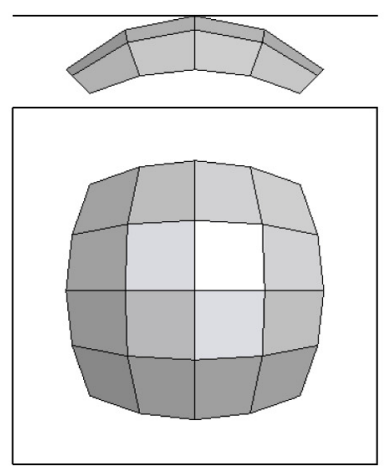

$\mathrm{b}$

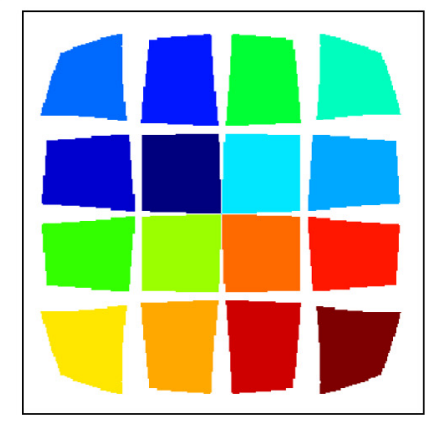

$\mathrm{c}$

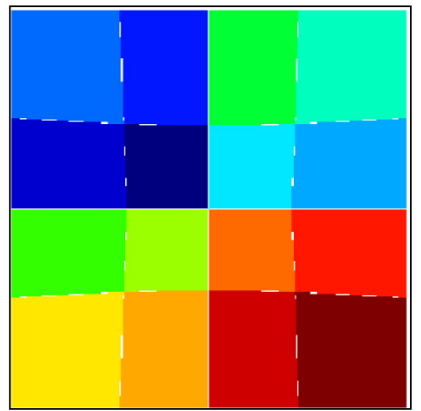

Fig. 13. Colour map of the slave points (flat surface) with projection on the spherical surface: (a) configuration of the surfaces (lateral and top views); (b) faceted description of the spherical surface; (c) smoothed description of the spherical surface.

segment presents its "normal projection" region, as shown in Fig. 12. However, often the assembly of the "normal projection" regions does not fill the neighbouring space completely, creating deadzones where no normal projection exists (no contact detected). Two types of blind spots can arise, internal and external. Slave nodes located in external blind spots are not detected before they penetrate the master surface, as shown in Fig. 12(a). On the other hand, slave nodes placed in internal blind spots (penetration into the master surface) are never detected, as shown in Fig. 12(b). The loss of history information is particularly important in frictional contact problems involving large sliding, since the tangential slip increment (Fig. 4) is defined through the local coordinates of the projection point in the last converged solution [16].

In order to highlight the improvements in the closest point projection when the master surface is smoothed with Nagata patches, a simple test case is presented. Two surfaces are involved, a flat (slave) surface and a convex spherical (master) surface, as shown in Fig. 13(a). The master surface is discretized by 16 quadrilateral finite elements, while a fine grid of points ( 300 divisions in each direction) is created over the square flat surface. The closest point projection is evaluated for each of these points to determine the corresponding master facet/patch. Considering the piecewise faceted description of the master surface, the colour map denoting the facets on which the slave points are projected with smallest normal gap is shown in Fig. 13(b). Some deadzones (white colour) arise near the common edges between finite elements, which are larger for points located further away from the convex surface, due to the pyramidal shape of the blind spots (see Fig. 12). On the other hand, the smoothing of the master surface with Nagata patches yields the colour map presented in Fig. 13(c), which represents the patches on which the slave points are projected with smallest normal gap. The blind spots observed for bilinear finite elements in Fig. 13(b) are strongly reduced using the surface smoothing approach. In fact, the zones of the slave surface without normal projection (white colour) are located in a very narrow range between the patches. Nevertheless, the deadzones are not completely eliminated using the Nagata patch interpolation. Therefore, the domain of each Nagata patch is slightly extended in all directions to cover the blind spots in the normal projection zone. The extension of the Nagata patch domain is performed incrementally up to a maximum of $2 \%$ increase in each direction, since the adoption of large values in the domain extension can lead to a switching between two adjacent patches, which degrades the local convergence. This increase only takes place if convergence is not reached within an admissible number of iterations. 


\subsection{Residual vectors and tangent matrices}

The residual vectors and tangent matrices of the developed contact elements are derived for the augmented Lagrangian method. The virtual work due to frictional contact was given in (17) for continuous problems, which takes the following form after grouping the derivatives (18)-(21):

$$
\delta W^{\mathrm{c}}=\left\{\begin{array}{l}
\int_{\bar{\gamma}_{\mathrm{c}}^{2 \bullet}} \hat{\lambda}_{\mathrm{n}} \delta g_{\mathrm{n}}+g_{\mathrm{n}} \delta \lambda_{\mathrm{n}}+\hat{\boldsymbol{\lambda}}_{\mathrm{t}} \cdot \delta \mathbf{g}_{\mathrm{t}}+\mathbf{g}_{\mathrm{t}} \cdot \delta \boldsymbol{\lambda}_{\mathrm{t}} d \gamma_{\mathrm{c}}^{2} \quad\left\|\hat{\boldsymbol{\lambda}}_{\mathrm{t}}\right\| \leqslant-\mu \hat{\lambda}_{\mathrm{n}}, \text { stick } \\
\int_{\bar{\gamma}_{\mathrm{c}}^{2 *}} \hat{\lambda}_{\mathrm{n}} \delta g_{\mathrm{n}}+g_{\mathrm{n}} \delta \lambda_{\mathrm{n}}-\mu \hat{\lambda}_{\mathrm{n}} \frac{\hat{\boldsymbol{\lambda}}_{\mathrm{t}}}{\left\|\hat{\boldsymbol{\lambda}}_{\mathrm{t}}\right\|} \cdot \delta \mathbf{g}_{\mathrm{t}}-\frac{1}{\varepsilon}\left(\boldsymbol{\lambda}_{\mathrm{t}}+\mu \hat{\lambda}_{\mathrm{n}} \frac{\hat{\boldsymbol{\lambda}}_{\mathrm{t}}}{\left\|\hat{\boldsymbol{\lambda}}_{\mathrm{t}}\right\|}\right) \cdot \delta \boldsymbol{\lambda}_{\mathrm{t}} d \gamma_{\mathrm{c}}^{2}\left\|\hat{\boldsymbol{\lambda}}_{\mathrm{t}}\right\|>-\mu \hat{\lambda}_{\mathrm{n}}, \text { slip } \\
\int_{\gamma_{\mathrm{c}}^{2} \backslash \bar{\gamma}_{\mathrm{c}}^{2}}-\frac{1}{\varepsilon} \lambda_{\mathrm{n}} \delta \lambda_{\mathrm{n}}-\frac{1}{\varepsilon} \boldsymbol{\lambda}_{\mathrm{t}} \cdot \delta \boldsymbol{\lambda}_{\mathrm{t}} d \gamma_{\mathrm{c}}^{2} \quad \hat{\lambda}_{\mathrm{n}}>0, \text { gap, }
\end{array}\right.
$$

where the contact surface is divided into three non-intersecting zones: $\bar{\gamma}_{\mathrm{c}}^{2 \bullet}$ stick zone, $\bar{\gamma}_{\mathrm{c}}^{2 *}$ slip zone and $\gamma_{\mathrm{c}}^{2} \backslash \bar{\gamma}_{\mathrm{c}}^{2}$ noncontact (gap) zone, representing the three possible contact statuses (stick, slip and gap). Thus, the integral contribution of the $i$ th contact element to the total virtual work can be written as:

$$
\delta W_{i}^{\mathrm{c}}=\int_{\partial \Omega_{i}^{2}}\left[\begin{array}{ll}
\mathbf{F}_{x} & \mathbf{F}_{\lambda}
\end{array}\right]^{\mathrm{T}} \cdot\left\{\begin{array}{l}
\delta \mathbf{x} \\
\delta \boldsymbol{\lambda}
\end{array}\right\} d \partial \Omega_{i}^{2},
$$

where the terms $\mathbf{F}_{x}$ and $\mathbf{F}_{\lambda}$ are vectors corresponding to forces acting on the virtual geometrical displacements $\delta \mathbf{x}$ and supplementary conditions acting on the virtual Lagrange multipliers $\delta \boldsymbol{\lambda}$ (contact forces), respectively [58]. In the framework of the finite element method, the first vector within the integral of Eq. (36) denotes the residual vector, while the second vector represents the degrees of freedom, which comprise both primal and dual variables.

Since the nodal normal vectors required for the Nagata interpolation are obtained by weighted average of adjacent facets (see Section 3.3), the patch geometry also depends on the nodes that form the edges attached to the master patch. Thus, the full linearization of the contact element comprises the nodes (three or four) associated with the master patch and all neighbouring ones coupled through the normal vectors [26]. Nevertheless, in order to preserve the local support of the new contact elements, the coupling with the neighbouring facets is neglected in this study, i.e. the variation of the nodal normal vectors is not taken into account. This simplification provides a banded structure for the global tangent matrix due to the low surface connectivity [17]. Indeed, the nonzero pattern of the global tangent matrix resulting from the surface smoothing procedure is identical to the one obtained with the faceted surface description. On the other hand, this linearization is not sufficient to ensure quadratic convergence in the iterative solution scheme. Considering the contact element composed by a quadrilateral Nagata patch (four master nodes), according to (35), the closed form of the residual vectors for stick, slip and gap contact statuses is given by:

$$
\begin{gathered}
\delta W_{\text {stick }}^{\mathrm{c}}=\left\{\begin{array}{c}
\hat{\lambda}_{\mathrm{n}} \mathbf{n}+\hat{\lambda}_{\mathrm{t}} \\
-w_{1}\left(\hat{\lambda}_{\mathrm{n}} \mathbf{n}+\hat{\lambda}_{\mathrm{t}}\right) \\
-w_{2}\left(\hat{\lambda}_{\mathrm{n}} \mathbf{n}+\hat{\boldsymbol{\lambda}}_{\mathrm{t}}\right) \\
-w_{3}\left(\hat{\lambda}_{\mathrm{n}} \mathbf{n}+\hat{\lambda}_{\mathrm{t}}\right) \\
-w_{4}\left(\hat{\lambda}_{\mathrm{n}} \mathbf{n}+\hat{\boldsymbol{\lambda}}_{\mathrm{t}}\right) \\
g_{n} \mathbf{n}+\mathbf{g}_{\mathrm{t}}
\end{array}\right\} \cdot\left\{\begin{array}{c}
\delta \mathbf{x}^{\mathrm{s}} \\
\delta \mathbf{x}_{1}^{\mathrm{m}} \\
\delta \mathbf{x}_{2}^{\mathrm{m}} \\
\delta \mathbf{x}_{3}^{\mathrm{m}} \\
\delta \mathbf{x}_{4}^{\mathrm{m}} \\
\delta \boldsymbol{\lambda}
\end{array}\right\}, \\
\delta W_{\text {slip }}^{\mathrm{c}}=\left\{\begin{array}{c}
\hat{\lambda}_{\mathrm{n}}(\mathbf{n}-\mu \mathbf{t}) \\
-w_{1}\left(\hat{\lambda}_{\mathrm{n}}(\mathbf{n}-\mu \mathbf{t})\right) \\
-w_{2}\left(\hat{\lambda}_{\mathrm{n}}(\mathbf{n}-\mu \mathbf{t})\right) \\
-w_{3}\left(\hat{\lambda}_{\mathrm{n}}(\mathbf{n}-\mu \mathbf{t})\right) \\
-w_{4}\left(\hat{\lambda}_{\mathrm{n}}(\mathbf{n}-\mu \mathbf{t})\right) \\
g_{n} \mathbf{n}-\left(\boldsymbol{\lambda}_{\mathrm{t}}+\mu \hat{\lambda}_{\mathrm{n}} \mathbf{t}\right) / \varepsilon
\end{array}\right\} \cdot\left\{\begin{array}{c}
\delta \mathbf{x}^{\mathrm{s}} \\
\delta \mathbf{x}_{1}^{\mathrm{m}} \\
\delta \mathbf{x}_{2}^{\mathrm{m}} \\
\delta \mathbf{x}_{3}^{\mathrm{m}} \\
\delta \mathbf{x}_{4}^{\mathrm{m}} \\
\delta \boldsymbol{\lambda}
\end{array}\right\},
\end{gathered}
$$




$$
\delta W_{g a p}^{\mathrm{c}}=\left\{\begin{array}{c}
\mathbf{0} \\
\mathbf{0} \\
\mathbf{0} \\
\mathbf{0} \\
\mathbf{0} \\
-\boldsymbol{\lambda} / \varepsilon
\end{array}\right\} \cdot\left\{\begin{array}{c}
\delta \mathbf{x}^{\mathrm{s}} \\
\delta \mathbf{x}_{1}^{\mathrm{m}} \\
\delta \mathbf{x}_{2}^{\mathrm{m}} \\
\delta \mathbf{x}_{3}^{\mathrm{m}} \\
\delta \mathbf{x}_{4}^{\mathrm{m}} \\
\delta \boldsymbol{\lambda}
\end{array}\right\}
$$

where the tangential slip direction involved in (38) is defined by:

$$
\mathbf{t}=\hat{\lambda}_{\mathrm{t}} /\left\|\hat{\lambda}_{\mathrm{t}}\right\|
$$

The action-reaction principle expressed by the momentum equilibrium at the contact interface is introduced by the weights $w_{i}$ associated to the master nodes. This means that the contact force arising in the slave node is distributed on the master nodes, according to the local coordinates of the closest point projection. In the present study, the weight associated to each master node is obtained from the partition of the Nagata patch area through the contact point coordinates. The expressions for the weight associated to each master node in case of quadrilateral Nagata patches takes the form:

$$
w_{1}=(1-\bar{\eta})(1-\bar{\zeta}), \quad w_{2}=\bar{\eta}(1-\bar{\zeta}), \quad w_{3}=\bar{\eta} \bar{\zeta} \quad \text { and } \quad w_{4}=\bar{\zeta}(1-\bar{\eta}),
$$

where the local coordinates of the contact point are calculated through the closest point projection. The residual vectors (37)-(39) for contact elements with triangular Nagata patch are obtained in a similar way, containing three lines for the master nodes and different associated weights, which can be found in [71].

The nonlinear and partially non-differentiable system of equations, resulting from the standard finite element assembly procedure of structural and contact elements, is solved using the generalized Newton method [10,14,20]. Then, the tangent contact matrix for each contact status needs to be computed, which does not takes into account the variation of the nodal normal vectors. The elemental contact Jacobian matrix is defined through the partial derivatives of the vectors $\mathbf{F}_{x}$ and $\mathbf{F}_{\lambda}$ present in (36), expressed by:

$$
\mathbf{J}^{\mathbf{c}}=\left[\begin{array}{cc}
\frac{\partial \mathbf{F}_{x}}{\partial \mathbf{x}} & \frac{\partial \mathbf{F}_{x}}{\partial \boldsymbol{\lambda}} \\
\frac{\partial \mathbf{F}_{\lambda}}{\partial \mathbf{x}} & \frac{\partial \mathbf{F}_{\lambda}}{\partial \boldsymbol{\lambda}}
\end{array}\right],
$$

which changes according with the contact status (gap, stick and slip).

Taking into account (37), the elemental Jacobian matrix of the contact element (four master nodes) for the stick contact status is given by:

$$
\mathbf{J}_{\text {stick }}^{\mathrm{c}}=\left[\begin{array}{cccccc}
\varepsilon \mathbf{I} & -w_{1} \varepsilon \mathbf{I} & -w_{2} \varepsilon \mathbf{I} & -w_{3} \varepsilon \mathbf{I} & -w_{4} \varepsilon \mathbf{I} & \mathbf{I} \\
-w_{1} \varepsilon \mathbf{I} & w_{1} w_{1} \varepsilon \mathbf{I} & w_{1} w_{2} \varepsilon \mathbf{I} & w_{1} w_{3} \varepsilon \mathbf{I} & w_{1} w_{4} \varepsilon \mathbf{I} & -w_{1} \mathbf{I} \\
-w_{2} \varepsilon \mathbf{I} & w_{2} w_{1} \varepsilon \mathbf{I} & w_{2} w_{2} \varepsilon \mathbf{I} & w_{2} w_{3} \varepsilon \mathbf{I} & w_{2} w_{4} \varepsilon \mathbf{I} & -w_{2} \mathbf{I} \\
-w_{3} \varepsilon \mathbf{I} & w_{3} w_{1} \varepsilon \mathbf{I} & w_{3} w_{2} \varepsilon \mathbf{I} & w_{3} w_{3} \varepsilon \mathbf{I} & w_{3} w_{4} \varepsilon \mathbf{I} & -w_{3} \mathbf{I} \\
-w_{4} \varepsilon \mathbf{I} & w_{4} w_{1} \varepsilon \mathbf{I} & w_{4} w_{2} \varepsilon \mathbf{I} & w_{4} w_{3} \varepsilon \mathbf{I} & w_{4} w_{4} \varepsilon \mathbf{I} & -w_{4} \mathbf{I} \\
\mathbf{I} & -w_{1} \mathbf{I} & -w_{2} \mathbf{I} & -w_{3} \mathbf{I} & -w_{4} \mathbf{I} & \mathbf{0}
\end{array}\right],
$$

where I is the second order identity tensor. Since the stick contact status imposes zero displacement between the slave node and the master surface [72], the local frame defined on the master surface is fixed in all Newton iterations within an increment. Thus, the solution can be considered path-independent, allowing to simplify the Jacobian matrix (symmetric) without affecting the convergence rate of the Newton method [73]. Concerning the slave node with slip status, the elemental contact Jacobian matrix developed by Heege and Alart [31] takes into account the curvature of the master surface. It is obtained from (38) considering the gradient of the unit normal vector at the contact point, 
stated as:

$$
\mathbf{J}_{\text {slip }}^{\mathrm{c}}=\left[\begin{array}{cccccc}
\varepsilon \mathbf{G} & -w_{1} \varepsilon \mathbf{G} & -w_{2} \varepsilon \mathbf{G} & -w_{3} \varepsilon \mathbf{G} & -w_{4} \varepsilon \mathbf{G} & \mathbf{M} \\
-w_{1} \varepsilon \mathbf{G} & w_{1} w_{1} \varepsilon \mathbf{G} & w_{1} w_{2} \varepsilon \mathbf{G} & w_{1} w_{3} \varepsilon \mathbf{G} & w_{1} w_{4} \varepsilon \mathbf{G} & -w_{1} \mathbf{M} \\
-w_{2} \varepsilon \mathbf{G} & w_{2} w_{1} \varepsilon \mathbf{G} & w_{2} w_{2} \varepsilon \mathbf{G} & w_{2} w_{3} \varepsilon \mathbf{G} & w_{2} w_{4} \varepsilon \mathbf{G} & -w_{2} \mathbf{M} \\
-w_{3} \varepsilon \mathbf{G} & w_{3} w_{1} \varepsilon \mathbf{G} & w_{3} w_{2} \varepsilon \mathbf{G} & w_{3} w_{3} \varepsilon \mathbf{G} & w_{3} w_{4} \varepsilon \mathbf{G} & -w_{3} \mathbf{M} \\
-w_{4} \varepsilon \mathbf{G} & w_{4} w_{1} \varepsilon \mathbf{G} & w_{4} w_{2} \varepsilon \mathbf{G} & w_{4} w_{3} \varepsilon \mathbf{G} & w_{4} w_{4} \varepsilon \mathbf{G} & -w_{4} \mathbf{M} \\
\mathbf{G} & -w_{1} \mathbf{G} & -w_{2} \mathbf{G} & -w_{3} \mathbf{G} & -w_{4} \mathbf{G} & \frac{1}{\varepsilon}(\mathbf{M}-\mathbf{I})
\end{array}\right],
$$

where the second order tensor $\mathbf{M}$, which is independent of the master surface curvature is defined as:

$$
\mathbf{M}=(\mathbf{n}-\mu \mathbf{t}) \otimes \mathbf{n}+\rho(\mathbf{I}-\mathbf{n} \otimes \mathbf{n}-\mathbf{t} \otimes \mathbf{t}), \quad \text { with } \rho=-\mu \hat{\lambda}_{\mathrm{n}} /\left\|\hat{\lambda}_{\mathrm{t}}\right\|,
$$

where $\rho \in[0,1]$ is a scaling factor. The supplementary curvature terms can be easily identified in the tensor $\mathbf{G}$, since they are coupled to the gradient of the normal vector:

$$
\begin{aligned}
\mathbf{G}= & (\mathbf{n}-\mu \mathbf{t}) \otimes \nabla_{\mathrm{x}} g_{\mathrm{n}}+\rho(\mathbf{I}-\mathbf{n} \otimes \mathbf{n}-\mathbf{t} \otimes \mathbf{t}) \\
& +\frac{1}{\varepsilon}\left\{(\mathbf{n}-\mu \mathbf{t}) \otimes \boldsymbol{\lambda}+\hat{\lambda}_{\mathrm{n}} \mathbf{I}-\rho\{\mathbf{n} \otimes(\boldsymbol{\lambda}+\varepsilon \mathbf{g})-((\boldsymbol{\lambda}+\varepsilon \mathbf{g}) \cdot \mathbf{n})(\mathbf{t} \otimes \mathbf{t}-\mathbf{I})\}\right\} \nabla_{\mathrm{x}} \mathbf{n} .
\end{aligned}
$$

Note that the elemental contact Jacobian matrix for the slip status (44) is non-symmetric due to the non-associativity of the Coulomb friction law and the curvature of the master surface [10,31]. Finally, in case of gap contact status, the elemental contact Jacobian matrix is easily obtained from (39), written as:

$$
\mathbf{J}_{\text {gap }}^{\mathbf{c}}=\left[\begin{array}{cccccc}
\mathbf{0} & \mathbf{0} & \mathbf{0} & \mathbf{0} & \mathbf{0} & \mathbf{0} \\
\mathbf{0} & \mathbf{0} & \mathbf{0} & \mathbf{0} & \mathbf{0} & \mathbf{0} \\
\mathbf{0} & \mathbf{0} & \mathbf{0} & \mathbf{0} & \mathbf{0} & \mathbf{0} \\
\mathbf{0} & \mathbf{0} & \mathbf{0} & \mathbf{0} & \mathbf{0} & \mathbf{0} \\
\mathbf{0} & \mathbf{0} & \mathbf{0} & \mathbf{0} & \mathbf{0} & \mathbf{0} \\
\mathbf{0} & \mathbf{0} & \mathbf{0} & \mathbf{0} & \mathbf{0} & -(1 / \varepsilon) \mathbf{I}
\end{array}\right],
$$

which is obviously a symmetric matrix.

The structure of the elemental Jacobian matrix (node-to-Nagata contact element) derived for each contact status (43), (44) and (47) can be represented by blocks. The first five rows/columns create a block comprising information related with the slave node and the corresponding quadrilateral Nagata patch (four master nodes). The last matrix row/column contains the gradient of the supplementary function, necessary to evaluate the nodal contact force. Thus, the penultimate row and column of the elemental Jacobian matrix are removed when the node-to-Nagata contact element is defined for a triangular Nagata patch (three master nodes). Note that the dimension of the elemental contact Jacobian matrices provided with the Nagata patch in the master surface description is exactly the same as in the classical node-to-segment contact element. Therefore, opposed to other surface smoothing methods that adopt Spline interpolation [17,23], the banded structure of the global tangent matrix is maintained as a result of adopting the local support of the Nagata interpolation.

\section{Numerical examples}

The proposed contact surface smoothing method was implemented in the in-house finite element code DD3IMP [74,75]. Four numerical examples were selected to assess the accuracy, robustness and performance of the new surface smoothing method based in the Nagata patch interpolation. All examples comprise the frictional contact between two deformable bodies involving large deformation and large sliding. The frictionless ironing problem is the first example considered, which was proposed by Sauer [76]. The second example involves the contact between two curved beams with elastoplastic material behaviour, which was introduced by Yang et al. [36] in the context of the mortar method. The third example comprises the sliding of a cylindrical contactor within a half-tube, firstly proposed by Krstulovic-Opara et al. [27]. The last example involves the rotation of two hollow concentric spheres with an initial 


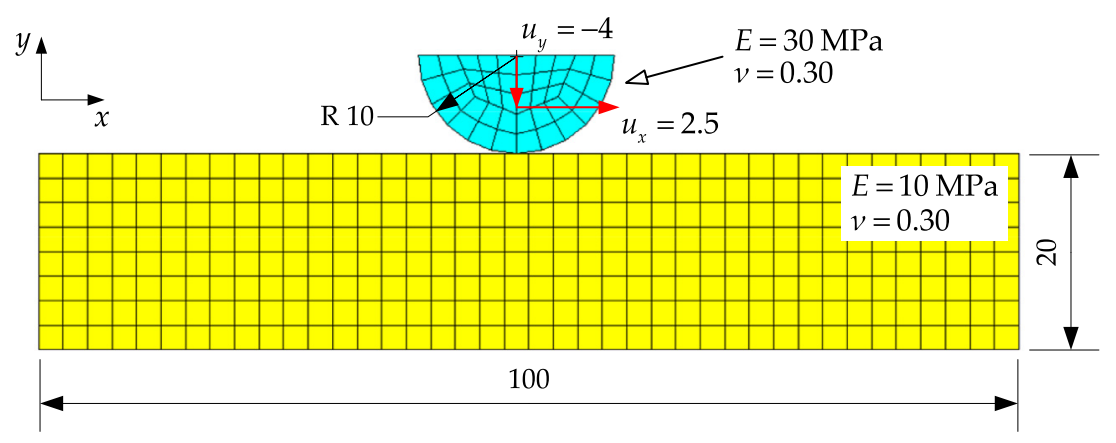

Fig. 14. Initial configuration of the frictionless ironing problem between deformable bodies (dimensions in $\mathrm{mm}$ ).

overlap, which is a severe benchmark for large sliding contact between spherical surfaces. The results obtained with the smoothed contact surface description are compared with the ones achieved through the standard faceted surface description, highlighting the advantages of the presented smoothing scheme. The numerical simulations were carried in a laptop equipped with an Intel ${ }^{\circledR}$ Core $^{\mathrm{TM}}$ i7-3630QM processor running at $2.4 \mathrm{GHz}$ and the Windows 8.1 (64-bits platform) operating system.

\subsection{Frictionless ironing problem}

The first example considers a deformable half-cylinder sliding over a deformable block, as shown in Fig. 14. This example is identical to the frictionless ironing problem reported by Sauer [76], and it is used to study the convergence behaviour of the surface smoothing method with mesh refinement. The half-cylinder (radius of $10 \mathrm{~mm}$ ) is pressed into the elastic block (dimensions $100 \times 20 \mathrm{~mm}$ ) and then moved horizontally. A vertical displacement of $4 \mathrm{~mm}$ is applied to the top of the cylinder, followed by a horizontal frictionless sliding of $2.5 \mathrm{~mm}$, as illustrated in Fig. 14. The bottom surface of the block is fixed. Both bodies are assumed elastic and isotropic, considering $E=30 \mathrm{MPa}$ and $v=0.30$ for the cylinder and $E=10 \mathrm{MPa}$ and $v=0.30$ for the block. Since the cylinder is 3 times stiffer than the block, it is chosen as master body. Both bodies are discretized with 8-node hexahedral finite elements, assuming plane strain conditions. Three different finite element meshes are considered, defined by the parameter $m \in\{3,4,5\}$, where the number of elements along the height of the block is given by $2^{m}(m=3$ is the mesh shown in Fig. 14). For a unitary increase of $m$, each element is subdivided into 4 smaller elements. Hence, the total number of finite elements composing the half-cylinder and the block is $21 \times 2^{2 m-5}$ and $5 \times 2^{2 m}$, respectively.

The comparison between faceted and smoothed contact surface description methods is presented in Fig. 15, for the frictionless ironing problem. The deformed configuration of the bodies at the end of sliding is slightly different due to the definition of the contact interface. The faceted description of the master surface yields the non-physical penetration of the master nodes into the slave body (block), as highlighted in Fig. 15(a). On the other hand, smoothing the curved surface of the cylinder with Nagata patches improves the definition of the contact interface and, consequently, the evaluation of the contact kinematics. In fact, the apparent penetration of the master nodes into the slave body is significantly reduced (see Fig. 15(b)). The distribution of the first stress invariant $I_{1}=\operatorname{tr}(\boldsymbol{\sigma})$ of the Cauchy stress tensor, at the end of sliding, is also presented in Fig. 15. The excessive penetration of the cylinder into the block reduces slightly the maximum (negative) value of the first stress invariant. Nevertheless, its distribution is similar using different approaches to describe the master contact surface.

The horizontal contact force evolution during the sliding, obtained with each surface description method (faceted and smoothed), is presented in Fig. 16(a) for three different finite element meshes. For the frictionless case considered in this example, the horizontal contact force $F_{x}$ should be zero. Nevertheless, the discontinuity of the surface normal vector field induced by the faceted contact description produces important non-physical oscillations in the contact force, as shown in Fig. 16(a). On the other hand, smoothing the master surface with Nagata patches yields insignificant oscillations in the contact force. In fact, adopting the surface smoothing method, the maximum value of $\left|F_{x}\right|$ is about $0.1 \mathrm{~N}$ for the coarsest mesh (846 nodes), while the standard bilinear surface representation provides a maximum force of approximately $0.5 \mathrm{~N}$ for the finer mesh (12,060 nodes). The convergence behaviour with the finite element mesh refinement is presented in Fig. 16(b) for both surface description methods, using the three nested meshes. The amplitude of the contact force oscillations decreases for increasing mesh refinement, as expected. For the same finite 
a

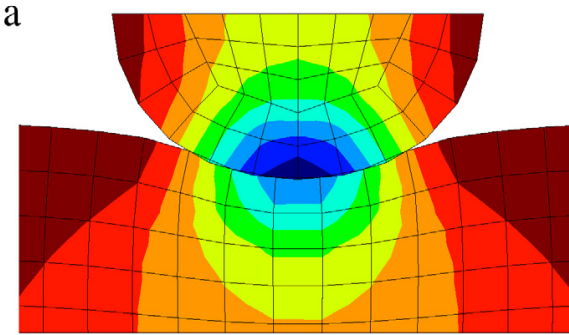

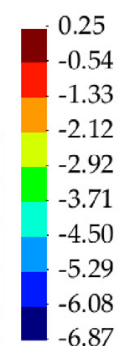

b

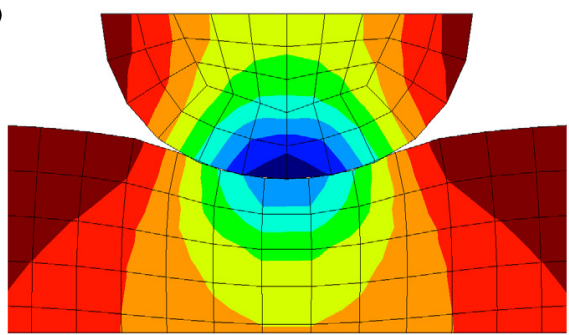

Fig. 15. Deformed configuration of the bodies involved in the ironing problem with stress invariant $I_{1}=\operatorname{tr}(\boldsymbol{\sigma})$ contours for: (a) faceted contact surface; (b) smoothed contact surface.
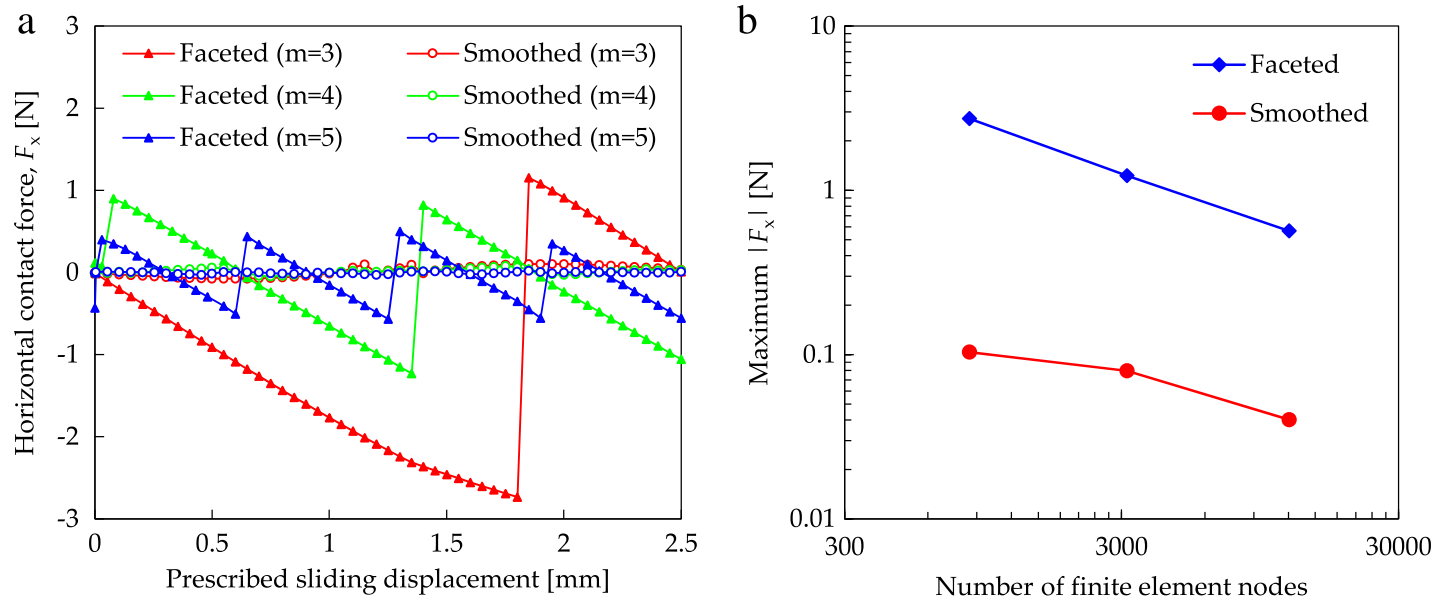

Fig. 16. Comparison between faceted and smoothed surface description methods in the frictionless ironing problem: (a) oscillations in the horizontal contact force evolution; (b) maximum value of horizontal contact force as function of the mesh refinement.

element discretization, the error in the horizontal contact force component is much lower for the smoothed than for the faceted description of the master surface.

\subsection{Contact between curved beams}

The second example was proposed by Yang et al. [36] and comprises contact between two curved beams (see Fig. 17) with large deformation and large sliding. This problem involves both material (elastoplastic behaviour) and geometric nonlinearities. The lower beam is fixed and the upper beam is subjected to a horizontal displacement of $31.5 \mathrm{~mm}$, as shown in Fig. 17. Both beams are modelled using an elastoplastic material with isotropic hardening. The elastic material properties are taken as $E=689.56 \mathrm{MPa}$ and $v=0.32$, while the plastic properties are given by the yield stress $\sigma_{0}=31 \mathrm{MPa}$ and the linear hardening rate $h=261.2 \mathrm{MPa}$. The two curved beams are discretized with 8-node hexahedral finite elements, as shown in Fig. 17, assuming plane strain conditions. The lower beam is defined as master body and the upper beam is considered as slave body. Both frictionless and frictional response is assumed between the beams. Two different values of friction coefficient are considered in this problem $\mu=0.3$ and $\mu=0.6$.

The configuration of the beams for $15 \mathrm{~mm}$ of prescribed displacement on the upper beam is presented in Fig. 18 for both frictionless and frictional cases, illustrating the deformed mesh and the nodal contact forces. In the frictionless case, the direction of the contact forces arising in the slave nodes is normal to the master contact surface (smoothed with Nagata patches). On the other hand, taking into account the friction at the contact interface, the direction of the nodal forces changes to produce a tangential component aligned with the slip direction, which is higher for larger values of friction coefficient, as shown in Fig. 18.

The evolution of the total reaction force in the $x$-direction, for the upper beam, is presented in Fig. 19 for the frictionless and frictional cases. Its amplitude increases for larger values of friction coefficient because the frictional force (tangential component) is close to the $x$-direction (see Fig. 18). Besides, the transition from negative to positive 


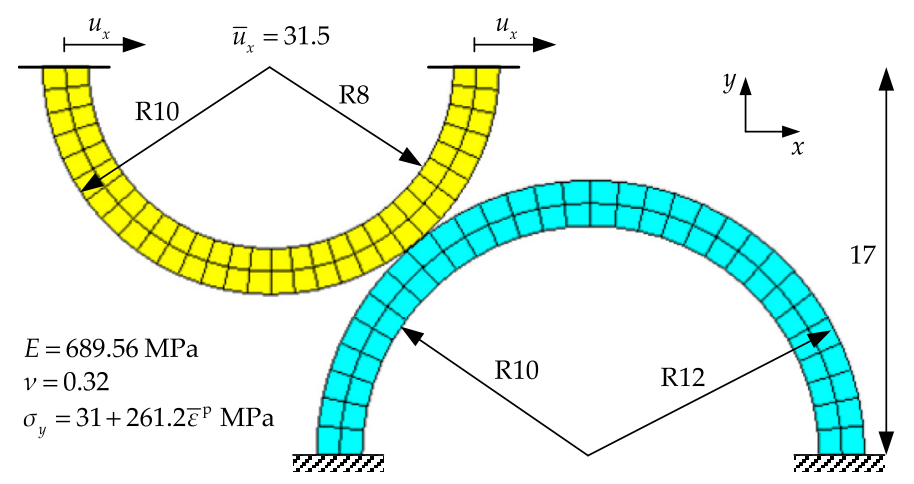

Fig. 17. Initial configuration and boundary conditions for the contact problem between two curved beams, including the finite element discretization (dimensions in $\mathrm{mm}$ ).

a

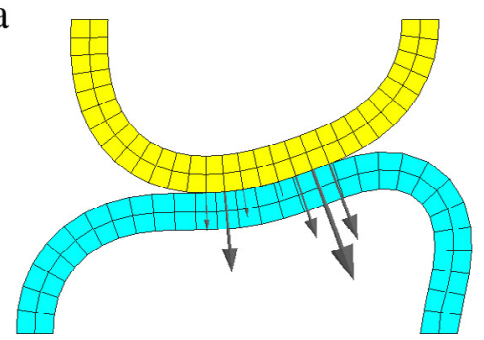

b

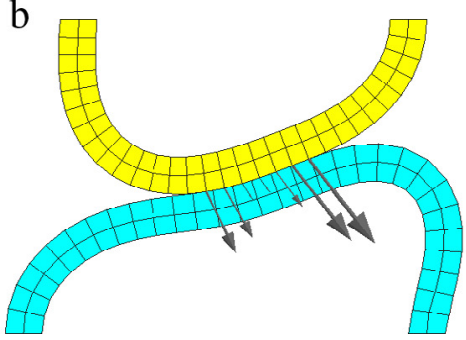

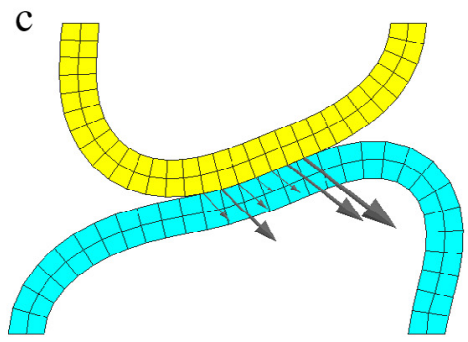

Fig. 18. Deformed configuration of the beams and nodal contact forces (size of the arrow proportional to the force magnitude) considering: (a) frictionless $(\mu=0.0)$; (b) frictional $(\mu=0.3)$; (c) frictional $(\mu=0.6)$.

values of force occurs later (higher displacement) and more suddenly for larger values of friction coefficient, as illustrated in Fig. 19. Adopting the faceted description of the master surface, the numerical simulation fails at $29 \mathrm{~mm}$ of prescribed displacement for the higher value of friction coefficient (see Fig. 19). This is related with the curved geometry of the master surface and the consequent deadzones in the normal projection, leading to severe problems in the contact detection procedure. The application of Nagata patches in the smoothing of the master surface allows to eliminate the non-physical oscillations observed in the contact force evolution. Moreover, the convergence is attained for all values of friction coefficient using 55 displacement increments. The evolution of the total reaction force in the $y$-direction, for the upper beam, is presented in Fig. 20 for the frictionless and frictional cases. In all cases, the force increases until attaining its maximum value and then decreases to zero. The horizontal displacement for which the maximum value of vertical force arises increases with the friction coefficient. On the other hand, the maximum value of reaction force in the $y$-direction increases slightly with the friction coefficient, as shown in Fig. 20.

The equivalent plastic strain contour plot is presented in Fig. 21 for both frictionless and frictional cases, at $15 \mathrm{~mm}$ of prescribed displacement on the upper beam. The plastic regions appear predominantly in the lower beam since its diameter is higher (see Fig. 17). Besides, the equivalent plastic strain is lower in the region near the contact area for higher values of friction coefficient, as illustrated in Fig. 21. This is related with the deformation mode of the lower curved beam, which changes from convex to concave for the frictionless case (Fig. 21(a)). On the other hand, considering the higher value of friction coefficient, the deformed configuration of the beam is approximately straight, as shown in Fig. 21(c). In fact, for the considered prescribed displacement $(15 \mathrm{~mm})$, the reaction force in the $y$-direction is lower for higher values of friction coefficient (see Fig. 20). The equivalent plastic strain distribution predicted with this model is very similar to the one obtained by Areias et al. [77].

\subsection{Cylindrical contactor sliding in a half-tube}

The third example involves a cylindrical contactor sliding in a half-tube (see Fig. 22). This benchmark was proposed by Krstulovic-Opara et al. [27] to assess the accuracy of the contact smoothing method in large sliding problems. The dimensions of the cylindrical contactor are $2 \times 2 \times 1 \mathrm{~mm}$ with a curvature radius of $3 \mathrm{~mm}$ in the contact surface. The 


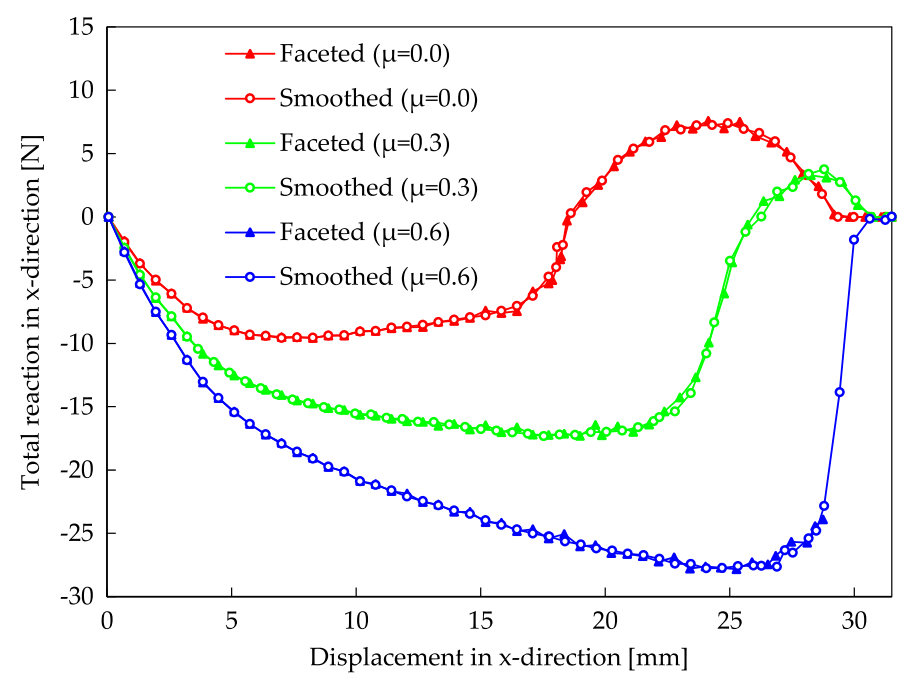

Fig. 19. Reaction force in the $x$-direction, for the upper beam, as function of its displacement for frictionless and frictional cases. Comparison between faceted and smoothed master surface descriptions.

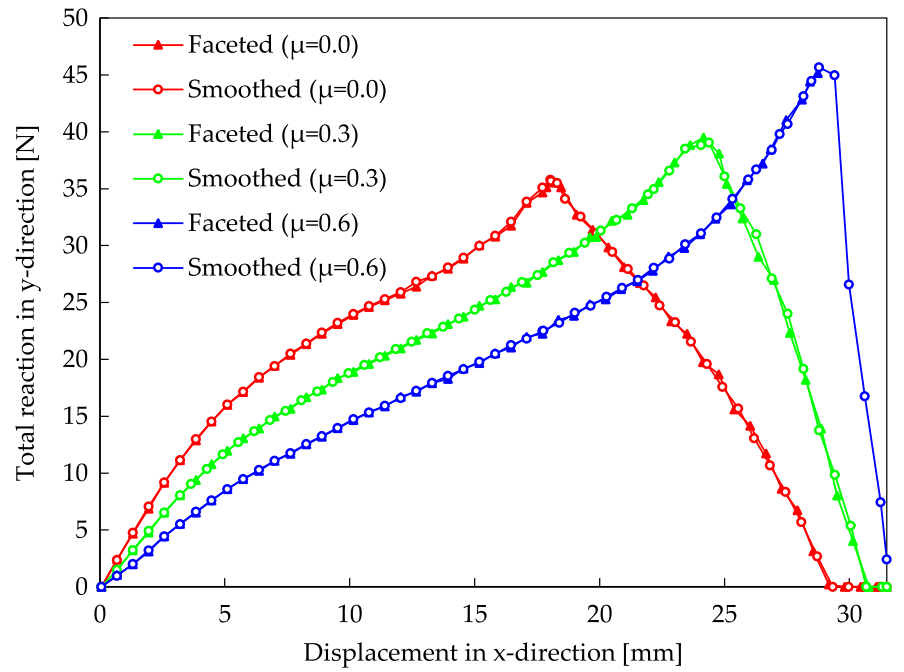

Fig. 20. Reaction force in the $y$-direction, for the upper beam, as function of its displacement for frictionless and frictional cases. Comparison between faceted and smoothed master surface descriptions.

half-tube has an interior radius of $3 \mathrm{~mm}$, thickness of $1 \mathrm{~mm}$ and $15 \mathrm{~mm}$ of length. Both bodies are modelled assuming elastic material response. The properties of the cylindrical contactor are taken as $E=10 \mathrm{MPa}$ and $v=0.30$, while the half-tube is 10 times stiffer than the contactor, so the Young's modulus and the Poisson's ratio are defined as $E=100 \mathrm{MPa}$ and $v=0.30$, respectively. The cylindrical contactor is chosen as slave body and the half-tube is considered the master body, defining the master contact surface. Both frictionless and frictional (Coulomb friction law with $\mu=0.15$ ) response is considered. The contactor is pressed into the half-tube (overlap of $\delta=0.05 \mathrm{~mm}$ ) and posteriorly moved $10 \mathrm{~mm}$ in the $x$-direction from the position illustrated in Fig. 22. The prescribed displacement is applied at the upper surface of the contactor and the half-tube is clamped in the $x O y$ (cutting) plane. Both bodies are discretized with 4-nodes tetrahedral finite elements, as shown in Fig. 22. Two distinct unstructured meshes are adopted, a coarse mesh composed by 2855 finite elements (Fig. 22(a)) and a fine mesh comprising 6327 finite elements (Fig. 22(b)).

Considering the coarse mesh, the evolution of the total reaction force in the $x$-direction is presented in Fig. 23 for both frictionless and frictional cases. The smoothing of the master contact surface using Nagata patch interpolation 


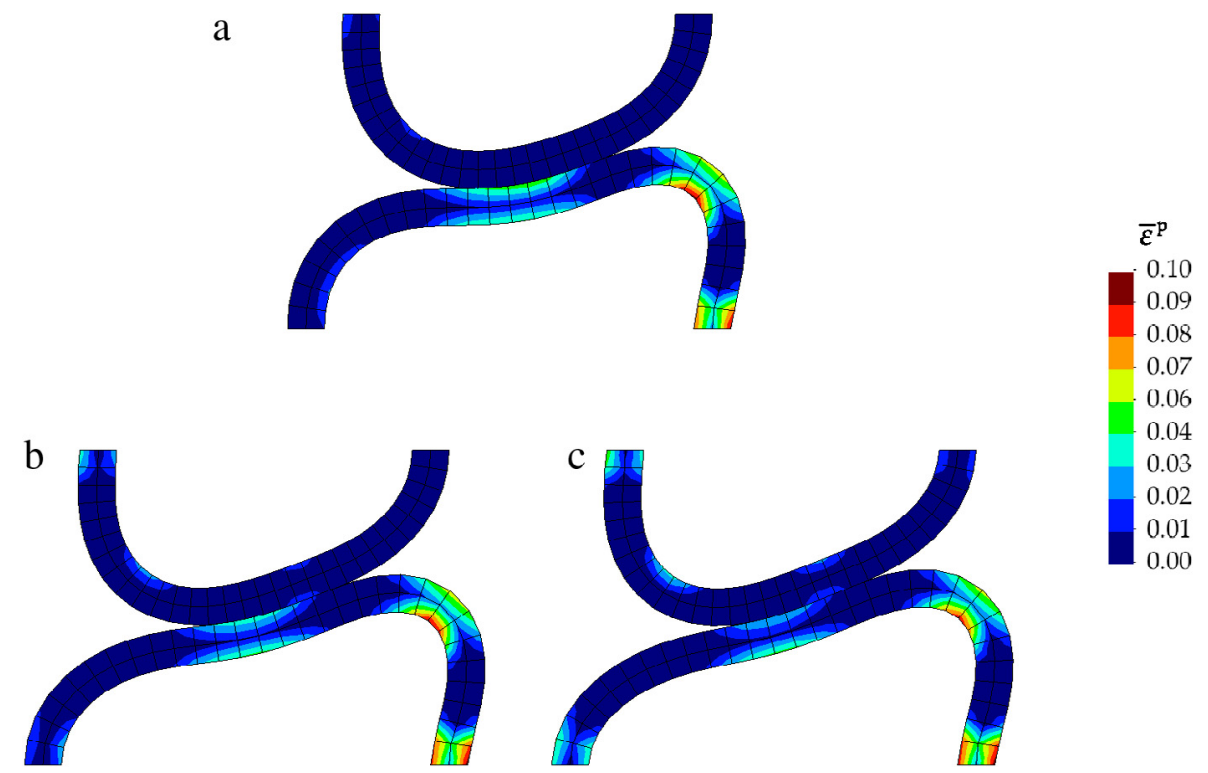

Fig. 21. Deformed configuration of the beams with equivalent plastic strain contours considering: (a) frictionless $(\mu=0.0)$; (b) frictional $(\mu=0.3)$; (c) frictional $(\mu=0.6)$.
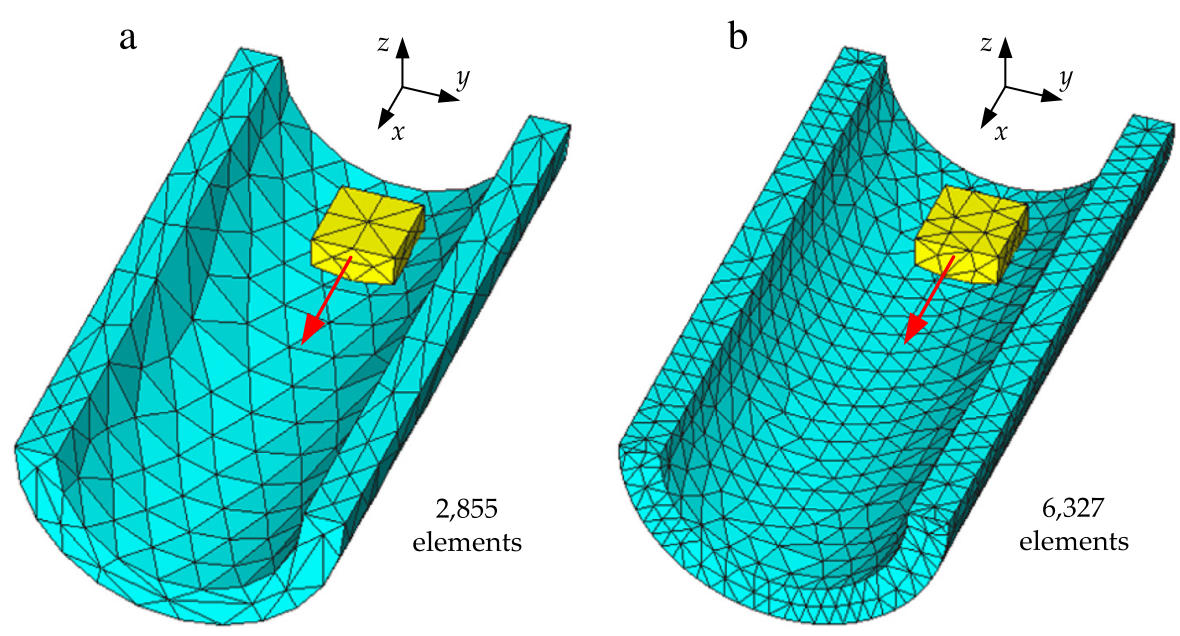

Fig. 22. Problem description for the cylindrical contactor sliding in a half-tube: (a) coarse mesh composed by 841 nodes; (b) fine mesh composed by 1746 nodes.

allows to eliminate the oscillations in the contact force induced by the piecewise bilinear interpolation of the master surface. In fact, the discretization of the tube using a relatively coarse mesh (Fig. 22(a)) leads to a bad approximation of the cylindrical geometry, which is reproduced in the contact force evolution. On the other hand, since the accuracy in the geometrical representation of the contact surface is significantly improved through the smoothing method (see Section 3.2), the oscillations in the contact force are strongly reduced or eliminated, as shown in Fig. 23. Considering the frictionless case, the total reaction contact force in $x$-direction (tangential component) is approximately zero when using Nagata patches to define the master contact surface.

The influence of the mesh refinement on the tangential reaction force evolution is presented in Fig. 24 for the frictional case, comparing the two approaches used to describe the master contact surface. As for the frictionless case, the oscillations observed in the contact force resulting from the faceted surface description are significantly reduced or eliminated with the surface smoothing scheme (see Fig. 24). Moreover, the accurate representation of the contact surface (smoothing method) leads to a decrease in the total reaction force in the $x$-direction, converging to 


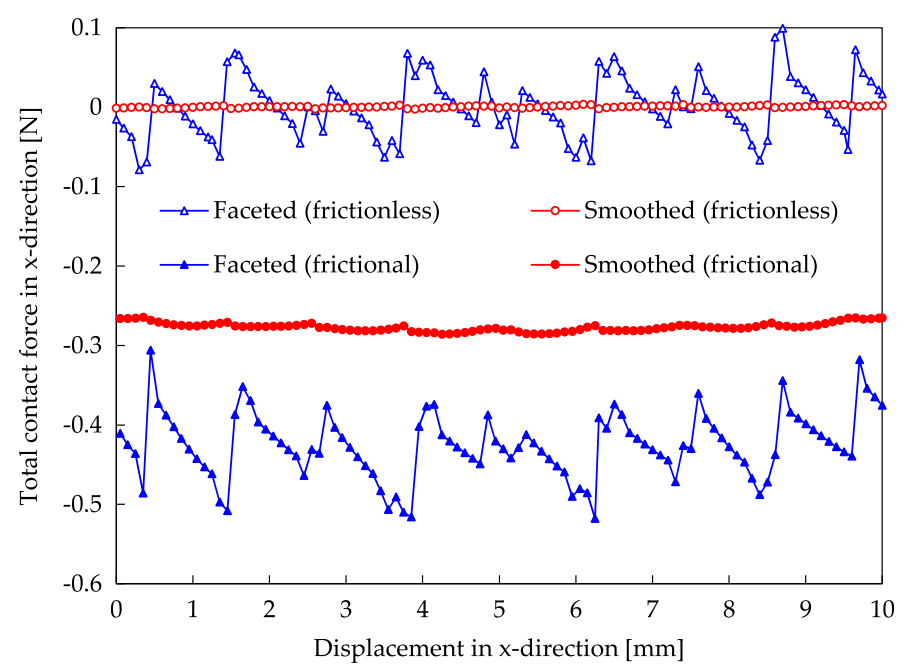

Fig. 23. Total tangential reaction force for frictionless and frictional cases, considering both faceted and smoothed descriptions of the contact master surface (coarse mesh).

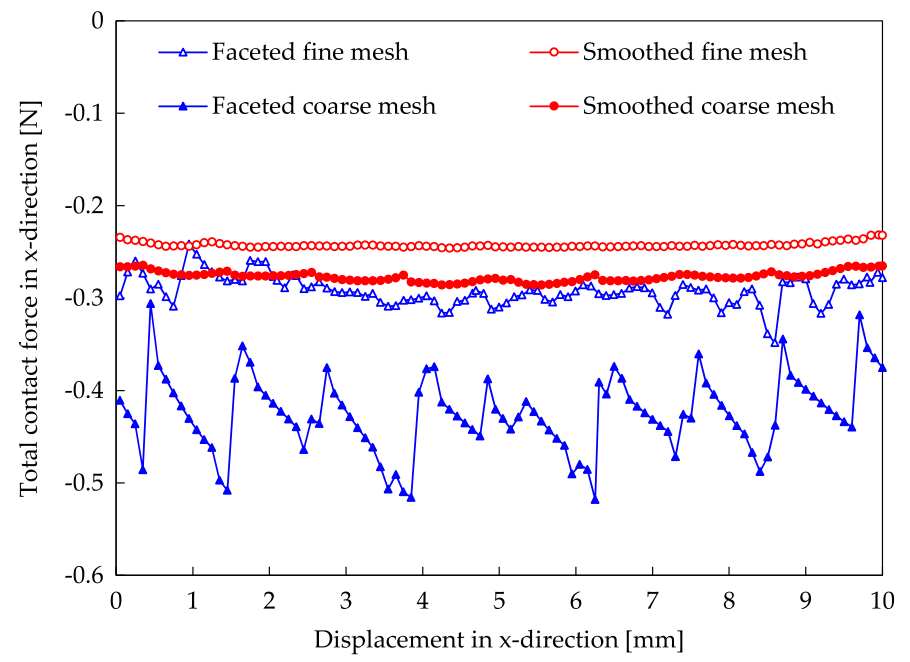

Fig. 24. Total tangential reaction force for both faceted and smoothed descriptions of the contact master surface (frictional case). Comparison between coarse and fine mesh.

the solution obtained with the fine mesh. In fact, the average value of contact force obtained with the fine mesh using the piecewise bilinear surface representation is similar to the one obtained with the coarse mesh using the surface smoothing approach, as illustrated in Fig. 24. The difference in the contact force evolution obtained with the coarse and fine meshes, using the contact surface smoothing method, results from the better description of the half-tube geometry, i.e. more finite elements in contact and non-contact zones.

The von Mises stress distribution in the contactor and half-tube, at the end of sliding, is presented in Fig. 25 for both finite element meshes, using different approaches to describe the master contact surface (faceted and smoothed). The application of Nagata patches leads to a reduction of the stress field value for both meshes studied. Indeed, the cylindrical geometry of the half-tube (master contact surface) defined by the piecewise bilinear interpolation is more interior than the smoothed surface, thus generating higher stress values, as shown in Fig. 25. On the other hand, the refinement of the finite element mesh used to describe the deformable bodies allows to predict more accurately the stress field. Moreover, the stress field obtained with the coarse mesh using the surface smoothing scheme (Fig. 25(b)) is similar to the one provided by the fine mesh with the surface smoothing (Fig. 25(d)). 

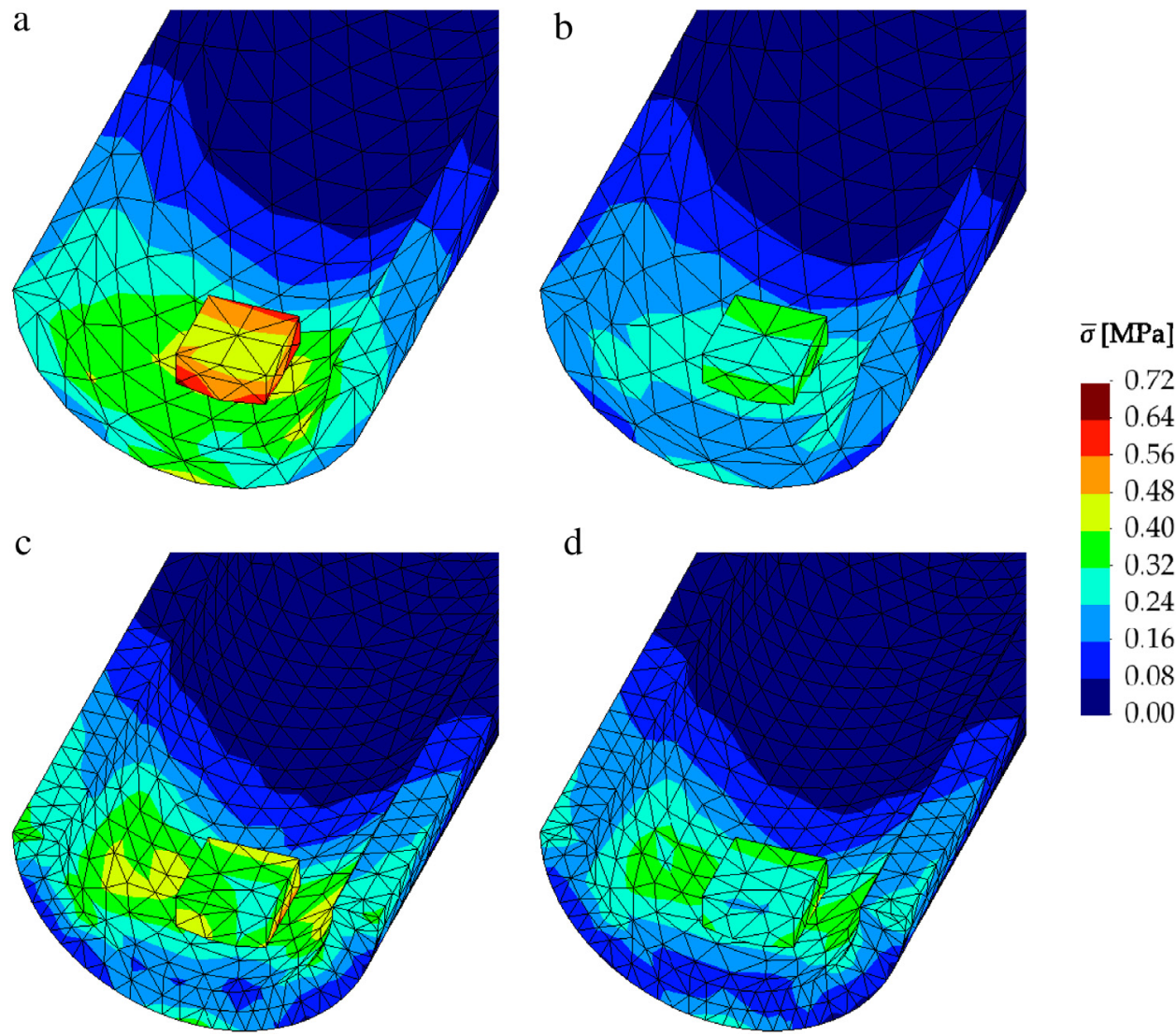

Fig. 25. von Mises stress distribution in the contactor and half-tube: (a) coarse mesh and faceted surface description; (b) coarse mesh and smoothed surface description; (c) fine mesh and faceted surface description; (d) fine mesh and smoothed surface description.

Table 1

Computational performance of the cylindrical contactor sliding in a half-tube problem for two distinct finite element meshes.

\begin{tabular}{llllll}
\hline & Coarse mesh & & Fine mesh & \\
\cline { 2 - 3 } \cline { 5 - 6 } & Faceted & Smoothed & & Faceted & Smoothed \\
\hline No. increments & 101 & 101 & 103 & 101 \\
Average no. iterations & 5.9 & 5.0 & 6.1 & 5.0 \\
Computational time (s) & 16.6 & 14.5 & 45.4 & 33.0 \\
\hline
\end{tabular}

The computational performance of this example is analysed in Table 1, considering only the sliding phase. The number of increments required to complete the simulation is similar for all models. Nevertheless, in order to overcome some convergence problems arising in the fine discretization with faceted surface description, the increment size is automatically reduced through the $r_{\min }$ strategy [78]. Indeed, the discontinuity of the surface normal vector field induced by the faceted description generates some convergence problems in the closest point projection (switching between two adjacent facets). Adopting the piecewise bilinear interpolation for the contact surface, the average number of iterations required to achieve convergence in each increment is approximately 6 , while the smoothing of the master surface requires an average of 5 iterations. Although the smoothing procedure requires additional linearization cost, the convergence rate is improved due to the quasi- $G^{1}$ continuity between patches, avoiding the above mentioned problems. Therefore, the application of the smoothing approach reduces the total computational time for both discretizations analysed. The reduction is higher for the fine mesh (approximately 27\%) because the total number of increments is lower (see Table 1). 


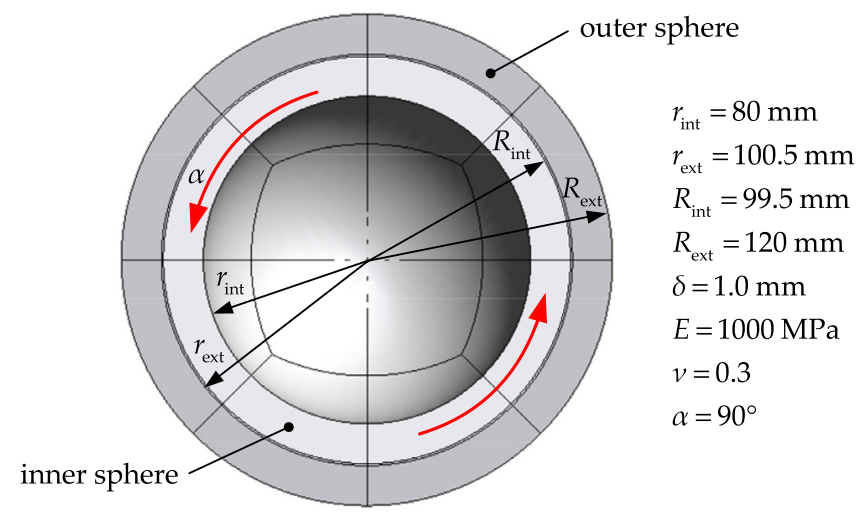

Fig. 26. Two concentric hollow spheres with initial overlap undergoing large sliding, including dimensions and material properties.
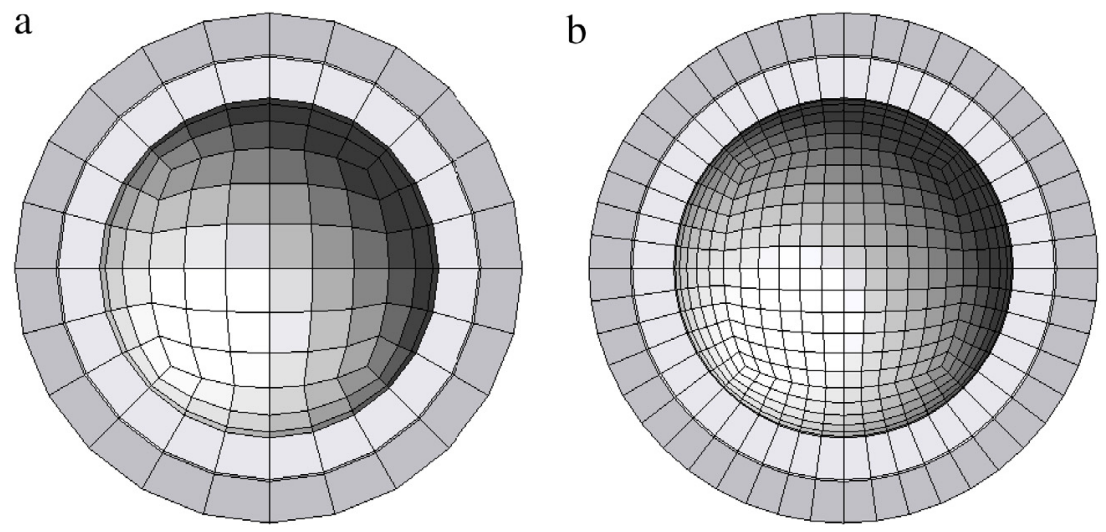

Fig. 27. Finite element mesh of the concentric hollow spheres: (a) coarse mesh with 484 nodes; (b) fine mesh with 1828 nodes.

\subsection{Rotation of hollow concentric spheres}

The last example is inspired in the contact problem proposed by Puso and Laursen [79], where two concentric spheres are pressurized internally and then rotated relative to each other. In the present study, the two concentric hollow spheres present an initial overlap $(\delta=1 \mathrm{~mm})$ and are rotated relative to each other producing large sliding, as shown in Fig. 26. The outer hollow sphere is fixed at its exterior radius, while the interior radius of the inner hollow sphere is rotated $90^{\circ}$ counter-clockwise. The main dimensions of the spheres are listed in Fig. 26, as well as the elastic material properties (identical for both spheres). Taking advantage of the symmetry conditions, only one half of the hollow spheres is modelled. Both spheres are discretized with 8-node hexahedral finite elements, adopting two different structured meshes, a coarse mesh composed by 216 finite elements (Fig. 27(a)) and a fine mesh comprising 864 finite elements (Fig. 27(b)). Both frictionless and frictional cases are considered.

The influence of the master-slave selection on the numerical results is analysed using the coarse mesh and considering the frictionless contact. Either the outer or the inner hollow sphere can be assigned as master body. The torque evolution (measured in the interior radius of the inner hollow sphere) as a function of the rotation angle is presented in Fig. 28, considering the two alternatives to define the master surface. The faceted description of the master surface leads to strong oscillations in the resulting torque, particularly when the outer hollow sphere is assigned as master body, as shown in Fig. 28. Since the mesh adopted for the spheres presents six finite elements to describe $90^{\circ}$ of the circumferential direction (see Fig. 27(a)), six waves are also generated in the torque evolution. On the other hand, the application of the Nagata patches in the smoothing of the master surface leads to a constant torque evolution being its value approximately zero (frictionless case), as shown in Fig. 28. In fact, for this case the numerical solution is somewhat independent of the selected master surface (outer or inner hollow sphere). A similar behaviour can be obtained 


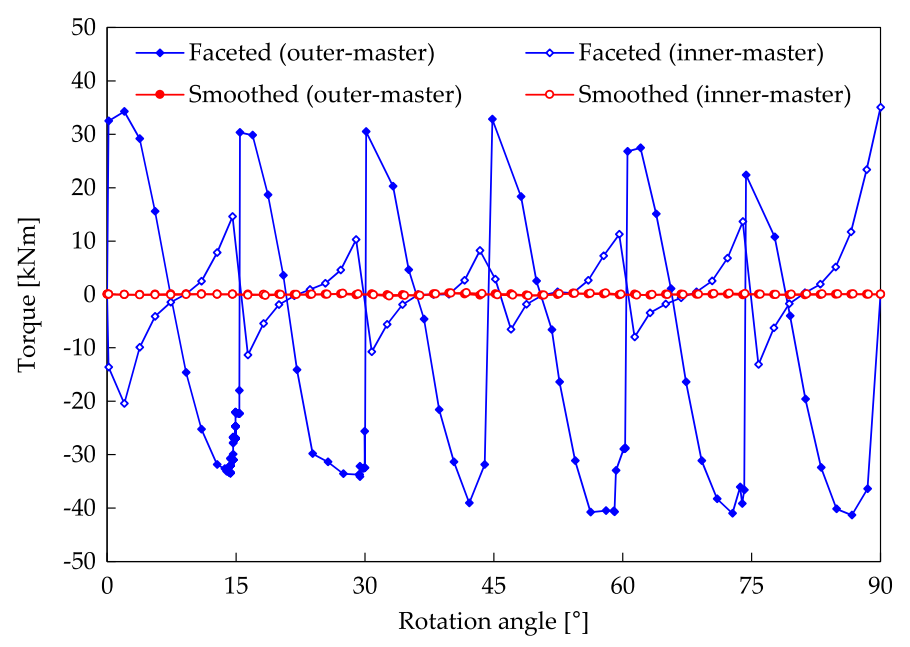

Fig. 28. Influence of the master-slave selection in the torque evolution (coarse mesh). Comparison between faceted and smoothed master surface descriptions.

with the two-half-pass formulation, recently proposed by Sauer and De Lorenzis [80], where the local equilibrium across the contact interface is not enforced $a$ prior due to the unbiased treatment of both contact surfaces.

The deformed configuration of the hollow spheres for a rotation angle of $7.5^{\circ}$ is presented in Fig. 29 considering the frictionless contact, highlighting the influence of the master-slave selection on the nodal contact forces. The numerical solution obtained with the piecewise bilinear interpolation of the master surface is strongly dependent on the choice of the master body. When the outer hollow sphere is assigned as master body, the enforcement of the impenetrability conditions at the slave nodes yields a large gap for the master nodes (see Fig. 29(a)), producing very high nodal contact forces. Alternatively, the selection of the inner hollow sphere as master body leads to the penetration of the master nodes into the slave body, as shown in Fig. 29(c). Thus, the resulting nodal contact forces are lower (the magnitude of the arrows depicted in figure was increased 5 times for visualization purposes). On the other hand, the smoothing of the master surface with Nagata patches allows the slave nodes to apparently "penetrate" the opposing master body, as highlighted in Fig. 29(b) and (d). Nevertheless, the deformed configuration obtained by switching the master and slave surfaces is identical for both situations, as well as the magnitude of the nodal contact forces, which is in accordance with the torque evolution presented in Fig. 28.

Considering friction at the contact interface between the spheres (Coulomb friction coefficient of $\mu=0.1$ ), the torque obtained with the coarse and fine meshes (see Fig. 27) is presented in Fig. 30 for both master surface description approaches. Taking into account the results obtained for the frictionless case (Fig. 28), the inner hollow sphere is defined as master body in the frictional case. The representation of the master surface using the piecewise bilinear interpolation leads to severe oscillations in the torque, particularly for the coarse mesh. The amplitude of the oscillations is reduced with the mesh refinement, while its frequency increases to double (due to the mesh refinement in the circumferential direction). Nevertheless, the torque value is always underestimated due to the admissible overlap of the master nodes into the slave body (see Fig. 29(c)), which reduces the contact pressure and, consequently, the frictional force. On the other hand, the smoothing of the master surface with Nagata patches yields a torque that increases from zero to a constant value (sliding of the contact interface), as shown in Fig. 30. Its constant value is approximately $33 \mathrm{kN} \mathrm{m}$, which increases approximately $2 \%$ with the mesh refinement.

The hollow spheres were also discretized with unstructured meshes of 8-node hexahedral (Fig. 31(a)) and 4-node tetrahedral (Fig. 31(b)) finite elements, to highlight the ability of the proposed surface smoothing method. The mesh defined by hexahedral finite elements is composed by 626 slave nodes (interior surface of the outer hollow sphere), while the mesh of tetrahedral elements contains 468 slave nodes. Concerning the number of facets defining the master contact surface (exterior surface of the inner hollow sphere), the discretization with hexahedral finite elements yields 371 quadrilateral finite elements, while the mesh of tetrahedral elements involves 456 triangular finite elements.

The torque evolution obtained with the unstructured meshes is presented in Fig. 32, which also compares faceted with smoothed master surface descriptions. The piecewise bilinear description of the master surface produces 
a
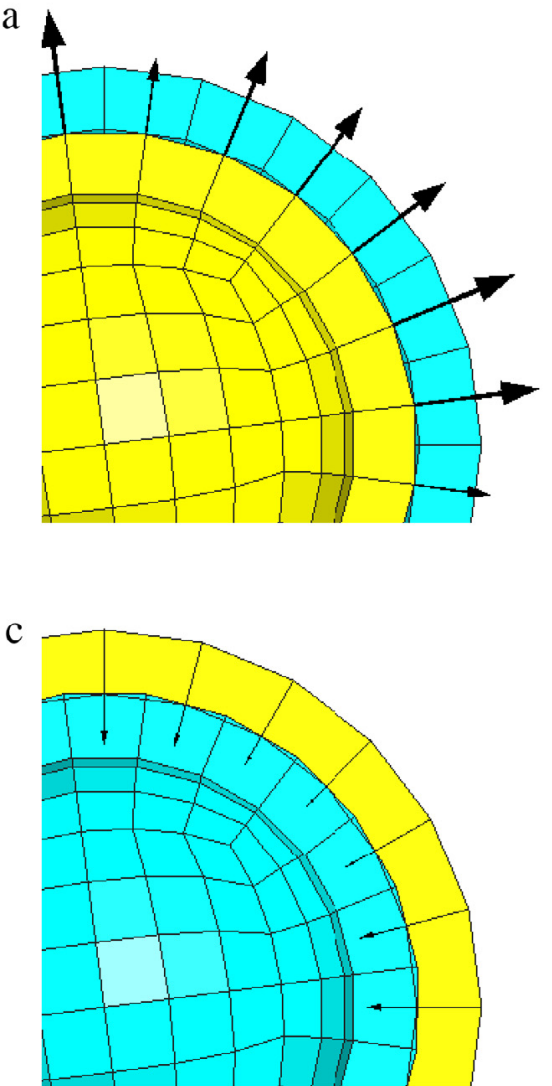

b

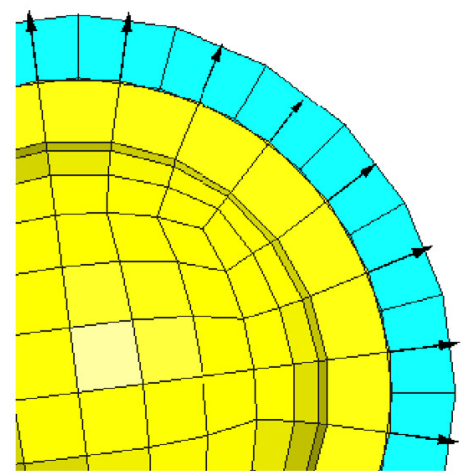

d

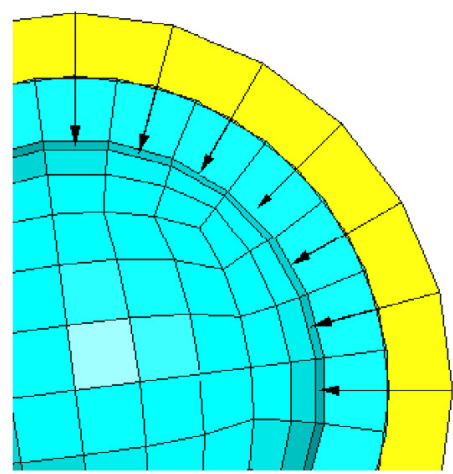

Fig. 29. Deformed configuration and nodal contact forces (size of the arrow proportional to the magnitude) for a rotation angle of $7.5^{\circ}:$ (a) outer sphere defined as master (faceted surface); (b) outer sphere defined as master (smoothed surface); (c) inner sphere defined as master (faceted surface) with arrows increased 5 times for visualization purposes; (d) inner sphere defined as master (smoothed surface).

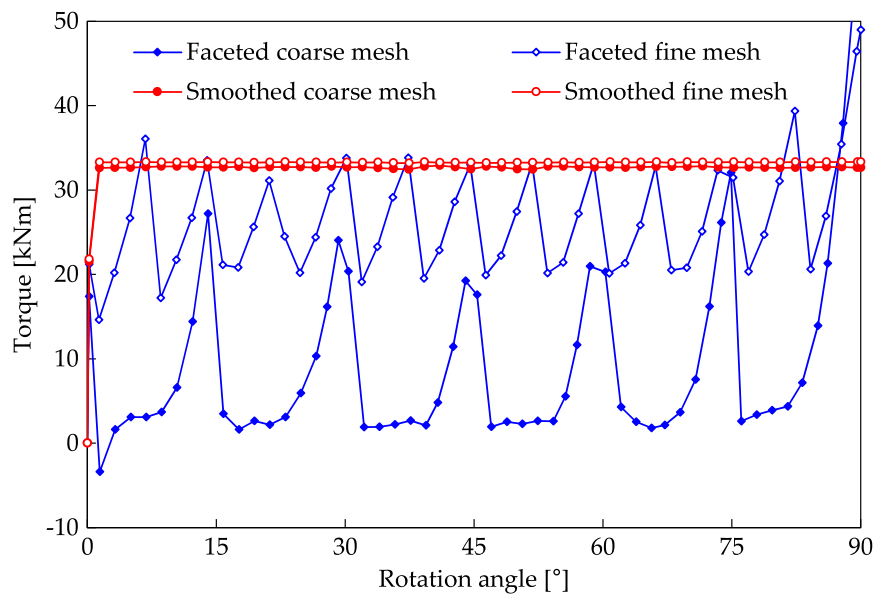

Fig. 30. Influence of the finite element mesh refinement in the torque evolution for the frictional case. Comparison between faceted and smoothed master surface descriptions.

oscillations in the torque evolution, which are smaller than in the case of the fine structured mesh (see Fig. 30). Furthermore, this surface description method yields a torque significantly lower than the one obtained with the smoothed description of the master surface, as shown in Fig. 32. This is related with the inaccurate representation 

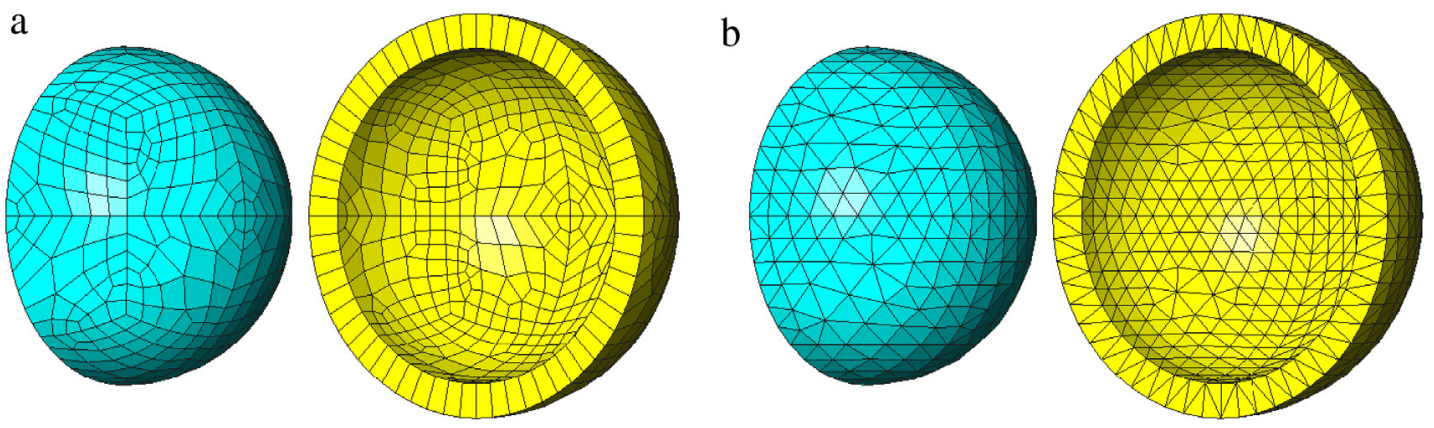

Fig. 31. Unstructured discretization of the hollow spheres using: (a) 8-node hexahedral finite elements (2004 nodes); (b) 4-node tetrahedral finite elements (1320 nodes).

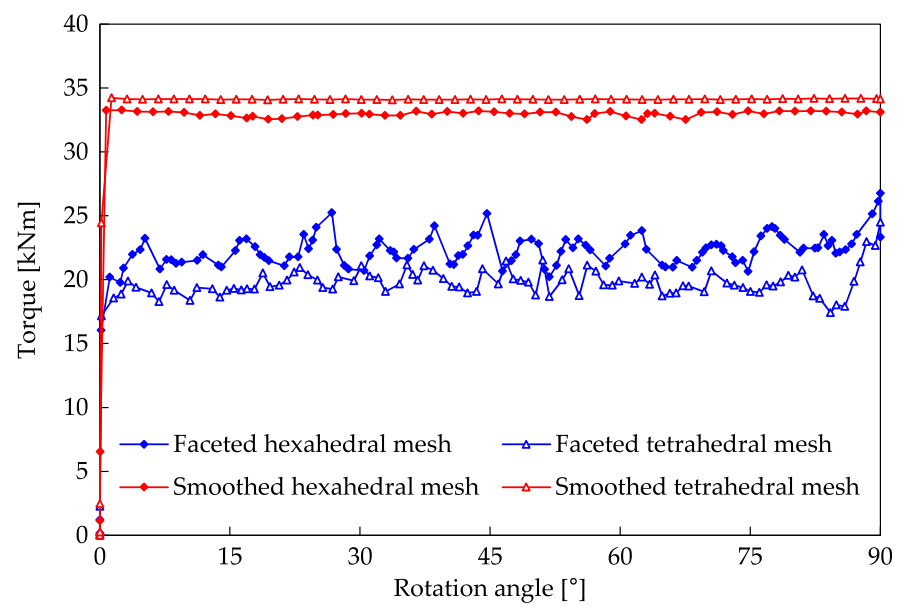

Fig. 32. Torque evolution with the rotation angle adopting unstructured finite element meshes. Comparison between faceted and smoothed master surface descriptions.

of the contact master surface (always interior to the smoothed surface) and, consequently, excessive penetration of the master nodes into the slave body. On the other hand, the adoption of the surface smoothing method leads to a torque evolution approximately constant ( $33 \mathrm{kN} \mathrm{m}$ ), which is similar to the one obtained with the structured meshes (Fig. 30). The effect of the surface bias resulting from the master-slave approach is less evident for unstructured meshes due to the arbitrarily distribution of the slave nodes on the master surface. Nevertheless, considering the outer hollow sphere defined as master body, the faceted description of the master surface provides a torque value significantly higher than applying the smoothing method.

The computational performance of the numerical simulations carried out using unstructured meshes is presented Table 2, for 8-node hexahedral and 4-node tetrahedral finite elements. The required number of increments is considerably higher in the faceted surface description than when using the surface smoothing method. Indeed, in order to overcome the convergence problems caused by the discontinuities in the surface normal vector field (typical of the faceted surface description), the increment size is automatically reduced. Concerning the number of iterations required in each increment, the average value is slightly lower when the master surface is smoothed with Nagata patches, as shown in Table 2. For this example, the computational time is predominantly dictated by the total number of increments. Consequently, applying the surface smoothing method reduces significantly the computational time. The reduction is about $40 \%$ using hexahedral finite elements in the discretization of the spheres and approximately $36 \%$ when the mesh is composed by tetrahedral finite elements. Since the number of nodes involved in the hexahedral finite element mesh is higher than in the tetrahedral finite element mesh (see Fig. 31), the computational time is substantially lower in the former mesh (see Table 2). 
Table 2

Computational performance of the two hollow concentric spheres problem considering unstructured finite element meshes.

\begin{tabular}{|c|c|c|c|c|}
\hline & \multicolumn{2}{|c|}{ Hexahedral finite elements } & \multicolumn{2}{|c|}{ Tetrahedral finite elements } \\
\hline & $\overline{\text { Faceted }}$ & Smoothed & $\overline{\text { Faceted }}$ & Smoothed \\
\hline No. increments & 114 & 58 & 96 & 54 \\
\hline Average no. iterations & 5.4 & 5.2 & 5.1 & 5.0 \\
\hline Computational time (s) & 167.7 & 99.3 & 35.7 & 22.7 \\
\hline
\end{tabular}

\section{Conclusions}

A new simple and effective 3D surface smoothing method has been presented for the numerical modelling of frictional contact problems between deformable bodies involving large sliding. The master surface is defined using Nagata patches to interpolate the low order finite element surface mesh at the nodes. The local support of the Nagata interpolation allows to smooth both regular and irregular surface meshes (arbitrary mesh topology), requiring only the surface normal vector at the nodes. These normal vectors are given by averaging the normal vectors of the facets adjacent to the master node. The smoothed surface is used to evaluate the kinematic contact variables, providing a description of contact phenomena closer to the physical reality. The smoothing method leads to significant improvements in the geometrical representation of the master surface in comparison with the piecewise bilinear surface representation. The original curvature is recovered with a relatively coarse mesh, ensuring quasi- $G^{1}$ continuity between patches. Therefore, the numerical simulation of contact problems based on implicit methods is considerably improved in terms of robustness, since the well-known convergence problems associated with the standard faceted description of the master surface $\left(C^{0}\right.$ continuity) are eliminated or strongly reduced.

Four numerical examples are used to highlight the advantages of the developed contact surface smoothing method. The results show a significant improvement in the accuracy, robustness and performance of the numerical simulations, when compared to the traditional piecewise faceted contact surface description. The non-physical oscillations in the contact forces evolution, resulting from the contact surface normal vector field discontinuities, are eliminated or strongly reduced with the surface smoothing approach, particularly for large sliding between curved contact surfaces. Furthermore, the smoothed master surface reduces the number of increments/equilibrium iterations required to perform the simulation due to superior convergence behaviour, i.e. the robustness is increased. Consequently, the computational time is reduced, improving the overall computational performance of the analysis.

\section{Acknowledgements}

The authors gratefully acknowledge the financial support of the Portuguese Foundation for Science and Technology (FCT) via the projects PTDC/EMS-TEC/1805/2012 and PEst-C/EME/UI0285/2013 and by FEDER funds through the program COMPETE-Programa Operacional Factores de Competitividade, under the project CENTRO-07-0224FEDER-002001 (MT4MOBI). The first author is also grateful to the FCT for the Ph.D. grant SFRH/BD/69140/2010.

\section{References}

[1] T.A. Laursen, Computational Contact and Impact Mechanics: Fundamentals of Modeling Interfacial Phenomena in Nonlinear Finite Element Analysis, Springer, Berlin, 2002.

[2] P. Wriggers, Computational Contact Mechanics, Springer, Berlin, 2006.

[3] J.T. Oden, E.B. Pires, Algorithms and numerical results for finite element approximations of contact problems with non-classical friction laws, Comput. Struct. 19 (1984) 137-147.

[4] J.O. Hallquist, G.L. Goudreau, D.J. Benson, Sliding interfaces with contact-impact in large-scale Lagrangian computations, Comput. Methods Appl. Mech. Engrg. 51 (1985) 107-137.

[5] D. Perić, D.R.J. Owen, Computational model for 3-D contact problems with friction based on the penalty method, Internat. J. Numer. Methods Engrg. 35 (1992) 1289-1309.

[6] P. Papadopoulos, R.L. Taylor, A mixed formulation for the finite element solution of contact problems, Comput. Methods Appl. Mech. Engrg. 94 (1992) 373-389.

[7] J.C. Simo, P. Wriggers, R.L. Taylor, A perturbed Lagrangian formulation for the finite element solution of contact problems, Comput. Methods Appl. Mech. Engrg. 50 (1985) 163-180. 
[8] P. Wriggers, J.C. Simo, A note on tangent stiffness for fully nonlinear contact problems, Commun. Appl. Numer. Methods 1 (1985) 199-203.

[9] P. Papadopoulos, J.M. Solberg, A Lagrange multiplier method for the finite element solution of frictionless contact problems, Math. Comput. Modelling 28 (1998) 373-384.

[10] P. Alart, A. Curnier, A mixed formulation for frictional contact problems prone to Newton like solution methods, Comput. Methods Appl. Mech. Engrg. 92 (1991) 353-375.

[11] G. DE Saxce, Z.Q. Feng, New inequality and functional for contact with friction: The implicit standard material approach, Mech. Struct. Mach. 19 (1991) 301-325.

[12] J.C. Simo, T.A. Laursen, An augmented Lagrangian treatment of contact problems involving friction, Comput. Struct. 42 (1992) $97-116$.

[13] B. Nour-Omid, P. Wriggers, A note on the optimum choice for penalty parameters, Commun. Appl. Numer. Methods 3 (1987) 581-585.

[14] Y. Renard, Generalized Newton's methods for the approximation and resolution of frictional contact problems in elasticity, Comput. Methods Appl. Mech. Engrg. 256 (2013) 38-55.

[15] A. Batailly, B. Magnain, N. Chevaugeon, A comparative study between two smoothing strategies for the simulation of contact with large sliding, Comput. Mech. 51 (2012) 581-601.

[16] P. Wriggers, L. Krstulovic-Opara, J. Korelc, Smooth C1-interpolations for two-dimensional frictional contact problems, Internat. J. Numer. Methods Engrg. 51 (2001) 1469-1495.

[17] V. Padmanabhan, T.A. Laursen, A framework for development of surface smoothing procedures in large deformation frictional contact analysis, Finite Elem. Anal. Des. 37 (2001) 173-198.

[18] M. Tur, E. Giner, F.J. Fuenmayor, P. Wriggers, 2D contact smooth formulation based on the mortar method, Comput. Methods Appl. Mech. Engrg. 247-248 (2012) 1-14.

[19] D.M. Neto, M.C. Oliveira, J.L. Alves, L.F. Menezes, Comparing faceted and smoothed tool surface descriptions in sheet metal forming simulation, Int. J. Mater. Form. 8 (2015) 549-565.

[20] G. Pietrzak, A. Curnier, Large deformation frictional contact mechanics: continuum formulation and augmented Lagrangian treatment, Comput. Methods Appl. Mech. Engrg. 177 (1999) 351-381.

[21] N. El-Abbasi, S.A. Meguid, A. Czekanski, On the modelling of smooth contact surfaces using cubic splines, Internat. J. Numer. Methods Engrg. 50 (2001) 953-967.

[22] M. Al-Dojayli, S.A. Meguid, Accurate modeling of contact using cubic splines, Finite Elem. Anal. Des. 38 (2002) $337-352$.

[23] M. Stadler, G.A. Holzapfel, J. Korelc, Cn continuous modelling of smooth contact surfaces using NURBS and application to 2D problems, Internat. J. Numer. Methods Engrg. 57 (2003) 2177-2203.

[24] C.J. Corbett, R.A. Sauer, NURBS-enriched contact finite elements, Comput. Methods Appl. Mech. Engrg. (2014).

[25] M. Stadler, G.A. Holzapfel, Subdivision schemes for smooth contact surfaces of arbitrary mesh topology in 3D, Internat. J. Numer. Methods Engrg. 60 (2004) 1161-1195.

[26] M.A. Puso, T.A. Laursen, A 3D contact smoothing method using Gregory patches, Internat. J. Numer. Methods Engrg. 54 (2002) 1161-1194.

[27] L. Krstulovic-Opara, P. Wriggers, J. Korelc, A C1-continuous formulation for 3D finite deformation frictional contact, Comput. Mech. 29 (2002) 27-42.

[28] T. Belytschko, W.J.T. Daniel, G. Ventura, A monolithic smoothing-gap algorithm for contact-impact based on the signed distance function, Internat. J. Numer. Methods Engrg. 55 (2002) 101-125.

[29] X. Qian, H. Yuan, M. Zhou, B. Zhang, A general 3D contact smoothing method based on radial point interpolation, J. Comput. Appl. Math. 257 (2014) 1-13.

[30] E. Hansson, A. Klarbring, Rigid contact modelled by CAD surface, Eng. Comput. 7 (1990) 344-348.

[31] A. Heege, P. Alart, A frictional contact element for strongly curved contact problems, Internat. J. Numer. Methods Engrg. 39 (1996) 165-184.

[32] J.H. Heegaard, A. Curnier, Geometric properties of 2D and 3D unilateral large slip contact operators, Comput. Methods Appl. Mech. Engrg. 131 (1996) 263-286.

[33] G. Zavarise, P. Wriggers, A segment-to-segment contact strategy, Math. Comput. Modelling 28 (1998) 497-515.

[34] T.W. McDevitt, T.A. Laursen, A mortar-finite element formulation for frictional contact problems, Internat. J. Numer. Methods Engrg. 48 (2000) $1525-1547$.

[35] M.A. Puso, T.A. Laursen, A mortar segment-to-segment frictional contact method for large deformations, Comput. Methods Appl. Mech. Engrg. 193 (2004) 4891-4913.

[36] B. Yang, T.A. Laursen, X. Meng, Two dimensional mortar contact methods for large deformation frictional sliding, Internat. J. Numer. Methods Engrg. 62 (2005) 1183-1225.

[37] K.A. Fischer, P. Wriggers, T.J.R. Hughes, J.T. Oden, M. Papadrakakis, Mortar based frictional contact formulation for higher order interpolations using the moving friction cone, Comput. Methods Appl. Mech. Engrg. 195 (2006) 5020-5036.

[38] T. Doca, F.M. Andrade Pires, J.M.A. Cesar de Sa, A frictional mortar contact approach for the analysis of large inelastic deformation problems, Int. J. Solids Struct. 51 (2014) 1697-1715.

[39] R. Taylor, P. Papadopoulos, On a patch test for contact problems in two dimensions, in: P. Wriggers, W. Wagner (Eds.), Comput. Methods Nonlinear Mech., Springer, 1991, pp. 690-702.

[40] N. El-Abbasi, K.-J. Bathe, Stability and patch test performance of contact discretizations and a new solution algorithm, Comput. Struct. 79 (2001) 1473-1486.

[41] G. Zavarise, L. De Lorenzis, The node-to-segment algorithm for 2D frictionless contact: Classical formulation and special cases, Comput. Methods Appl. Mech. Engrg. 198 (2009) 3428-3451.

[42] İ. Temizer, P. Wriggers, T.J.R. Hughes, Three-dimensional mortar-based frictional contact treatment in isogeometric analysis with NURBS, Comput. Methods Appl. Mech. Engrg. 209-212 (2012) 115-128.

[43] L. De Lorenzis, İ. Temizer, P. Wriggers, G. Zavarise, A large deformation frictional contact formulation using NURBS-based isogeometric analysis, Internat. J. Numer. Methods Engrg. 87 (2011) 1278-1300. 
[44] L. De Lorenzis, P. Wriggers, T.J.R. Hughes, Isogeometric contact: a review, GAMM-Mitt. 37 (2014) 85-123.

[45] T.J.R. Hughes, J.A. Cottrell, Y. Bazilevs, Isogeometric analysis: CAD, finite elements, NURBS, exact geometry and mesh refinement, Comput. Methods Appl. Mech. Engrg. 194 (2005) 4135-4195.

[46] J. Lu, Isogeometric contact analysis: Geometric basis and formulation for frictionless contact, Comput. Methods Appl. Mech. Engrg. 200 (2011) 726-741.

[47] J. Schöberl, NETGEN_-An advancing front 2D/3D-mesh generator based on abstract rules, Comput. Vis. Sci. 1 (1997) $41-52$.

[48] I. Guskov, A. Khodakovsky, P. Schröder, W. Sweldens, Hybrid meshes: Multiresolution using regular and irregular refinemen, in: Proc. Eighteenth Annu. Symp. Comput. Geom.-SCG'02, ACM Press, New York, New York, USA, 2002, pp. 264-272.

[49] T. Nagata, Simple local interpolation of surfaces using normal vectors, Comput. Aided Geom. Design 22 (2005) $327-347$.

[50] T. Hama, T. Nagata, C. Teodosiu, A. Makinouchi, H. Takuda, Finite-element simulation of springback in sheet metal forming using local interpolation for tool surfaces, Int. J. Mech. Sci. 50 (2008) 175-192.

[51] M. Hachani, L. Fourment, A smoothing procedure based on quasi-C1 interpolation for 3D contact mechanics with applications to metal forming, Comput. Struct. 128 (2013) 1-13.

[52] D.M. Neto, M.C. Oliveira, L.F. Menezes, J.L. Alves, Applying Nagata patches to smooth discretized surfaces used in 3D frictional contact problems, Comput. Methods Appl. Mech. Engrg. 271 (2014) 296-320.

[53] T.A. Laursen, J.C. Simo, A continuum-based finite element formulation for the implicit solution of multibody, large deformation-frictional contact problems, Internat. J. Numer. Methods Engrg. 36 (1993) 3451-3485.

[54] A. Konyukhov, K. Schweizerhof, On the solvability of closest point projection procedures in contact analysis: Analysis and solution strategy for surfaces of arbitrary geometry, Comput. Methods Appl. Mech. Engrg. 197 (2008) 3045-3056.

[55] A. Curnier, A theory of friction, Int. J. Solids Struct. 20 (1984) 637-647.

[56] C. Agelet de Saracibar, A new frictional time integration algorithm for large slip multi-body frictional contact problems, Comput. Methods Appl. Mech. Engrg. 142 (1997) 303-334.

[57] J.-H. Heegaard, A. Curnier, An augmented Lagrangian method for discrete large-slip contact problems, Internat. J. Numer. Methods Engrg. 36 (1993) 569-593.

[58] V.A. Yastrebov, Numerical Methods in Contact Mechanics, John Wiley \& Sons, 2013.

[59] J. Lengiewicz, J. Korelc, S. Stupkiewicz, Automation of finite element formulations for large deformation contact problems, Internat. J. Numer. Methods Engrg. 85 (2011) 1252-1279.

[60] S. Jin, R.R. Lewis, D. West, A comparison of algorithms for vertex normal computation, Vis. Comput. 21 (2005) 71-82.

[61] D.M. Neto, M.C. Oliveira, L.F. Menezes, J.L. Alves, Improving Nagata patch interpolation applied for tool surface description in sheet metal forming simulation, Comput. Aided Des. 45 (2013) 639-656.

[62] D. Chamoret, P. Saillard, A. Rassineux, J.-M. Bergheau, New smoothing procedures in contact mechanics, J. Comput. Appl. Math. 168 (2004) 107-116.

[63] M. Boschiroli, C. Fünfzig, L. Romani, G. Albrecht, A comparison of local parametric C0 Bézier interpolants for triangular meshes, Comput. Graph. 35 (2011) 20-34.

[64] D.M. Neto, M.C. Oliveira, L.F. Menezes, J.L. Alves, Nagata patch interpolation using surface normal vectors evaluated from the IGES file, Finite Elem. Anal. Des. 72 (2013) 35-46.

[65] D.L. Page, Y. Sun, A.F. Koschan, J. Paik, M.A. Abidi, Normal vector voting: Crease detection and curvature estimation on large, noisy meshes, Graph. Models 64 (2002) 199-229.

[66] H. Gouraud, Continuous shading of curved surfaces, IEEE Trans. Comput. C-20 (1971) 623-629.

[67] G. Thürmer, C.A. Wüthrich, Computing vertex normals from polygonal facets, J. Graph. Tools 3 (1998) 43-46.

[68] N. Max, Weights for computing vertex normals from facet normals, J. Graph. Tools 4 (1999) 1-6.

[69] D.J. Benson, J.O. Hallquist, A single surface contact algorithm for the post-buckling analysis of shell structures, Comput. Methods Appl. Mech. Engrg. 78 (1990) 141-163.

[70] A. Konyukhov, K. Schweizerhof, Covariant description for frictional contact problems, Comput. Mech. 35 (2005) 190-213.

[71] D.M. Neto, Numerical simulation of frictional contact problems using nagata patches in surface smoothing, University of Coimbra, 2014.

[72] P. Alart, A. Heege, Consistent tangent matrices of curved contact operators involving anisotropic friction, Rev. Européenne Élém. Finis 4 (1995) 183-207.

[73] M.C. Oliveira, J.L. Alves, L.F. Menezes, Algorithms and strategies for treatment of large deformation frictional contact in the numerical simulation of deep drawing process, Arch. Comput. Methods Eng. 15 (2008) 113-162.

[74] L.F. Menezes, C. Teodosiu, Three-dimensional numerical simulation of the deep-drawing process using solid finite elements, J. Mater. Process. Technol. 97 (2000) 100-106.

[75] L.F. Menezes, D.M. Neto, M.C. Oliveira, J.L. Alves, Improving computational performance through HPC techniques: case study using DD3IMP in-house code, AIP Conf. Proc. 1353 (2011) 1220-1225.

[76] R.A. Sauer, Local finite element enrichment strategies for 2D contact computations and a corresponding post-processing scheme, Comput. Mech. 52 (2013) 301-319.

[77] P. Areias, T. Rabczuk, F.J.M. Queirós de Melo, J. César de Sá, Coulomb frictional contact by explicit projection in the cone for finite displacement quasi-static problems, Comput. Mech. 55 (2014) 57-72.

[78] M.C. Oliveira, L.F. Menezes, Automatic correction of the time step in implicit simulations of the stamping process, Finite Elem. Anal. Des. 40 (2004) 1995-2010.

[79] M.A. Puso, T.A. Laursen, A mortar segment-to-segment contact method for large deformation solid mechanics, Comput. Methods Appl. Mech. Engrg. 193 (2004) 601-629.

[80] R.A. Sauer, L. De Lorenzis, An unbiased computational contact formulation for 3D friction, Internat. J. Numer. Methods Engrg. 101 (2015) 251-280. 